Feng Wang

Li Zhou

Zao-jian Zou

Ming Song

Yang Wang

Yi Liu

http://dx.doi.org/10.21278/brod70306

ISSN 0007-215X

eISSN 1845-5859

\title{
STUDY OF CONTINUOUS ICEBREAKING PROCESS WITH COHESIVE ELEMENT METHOD
}

UDC 629.5.016.5:629.561.5

Original scientific paper

\begin{abstract}
Summary
Accurate simulation of the continuous icebreaking process in level ice is crucial for the design of icebreakers. The crushing and bending failures of ice sheet, as well as the rotating, sliding and accumulating of ice cusps broken from the ice sheet constitute a complex system for the icebreaking process. In this paper, cohesive element method is combined with an elastoplastic softening constitutive model to simulate continuous icebreaking process in level ice. Firstly, the elastoplastic softening constitutive model in modelling ice local crushing is calibrated by simulating the ice cone crushing tests. Three different softening laws are proposed and their effects on simulation results are evaluated by comparing with the experimental data. Then, the continuous icebreaking process in level ice is simulated by cohesive element method. The regular tri-prism mesh is applied to ice bulk elements to realize the random propagation of crack. The mesh dependency study is carried out, and the simulation results are validated by comparing with model test results in both of time domain and frequency domain. The ice failure patterns during continuous icebreaking process are also compared between the simulated and experimental results. Finally, the influences of ship velocity on ice resistance and ice failure patterns are investigated by numerical methods and semi-empirical formulas.
\end{abstract}

Key words: $\quad$ icebreaking; cohesive element; constitutive law; ice resistance; Ice failure patterns; ship velocity

\section{Introduction}

With the global warming, ice cap in the Arctic has been shrinking year by year. Consequently, there will be more and more voyages across the Arctic. Accurate prediction of ice resistance on the vessels is necessary for their design and safe navigation in the icecovered waters. When a ship is traveling in level ice, the ice sheet will experience breaking failure, which mainly includes local crushing and bending failures. The generated ice cusps rotate, slide and accumulate around the hull, which leads to additional ice resistance on the 
ship. Based on the research by Croasdale and Cammaert [1], the proportion of accumulation forces in the total ice loads may reach up to $70 \%$. Thus, it is important to simulate the whole ship-ice interaction process for a better prediction of ice resistance.

Since the mechanism of icebreaking process has not been fully understood yet, many researchers have applied empirical formulae to predict ice resistance since 1970 [2-4]. In these formulas, the empirical coefficients were derived by fitting the data from the model tests and full-scale trials. Due to the limited data, the expressions contain only a few empirical constants and their applications are limited. The semi-empirical approach with theoretically derived mechanical formulae can consider more details of ice-structure interaction. Lindqvist [5] summed up the sea trials in the Bay of Bothnia and divided ice resistance into crushing, bending and submersion components. Lindstrom [6] adopted fully derived mechanical formulas and manifested the physical concepts of ice-hull interaction. However, Lindstrom's model only considered the bending failure of ice. Based on a series of full-scale tests in Baltic Sea, Riska et al. [7] presented a formula for ice resistance by improving the empirical formulas proposed by Lindqvist [5]. The Riska formula considered ice resistance as a function of ship main dimensions.

With the rapid promotion of computational power, discrete element method (DEM) and finite element method (FEM) have been widely used to model the ice-structure interactions. Paavilainen et al. [8] applied DEM to simulate ice pile-up process against an inclined structure. Zhan et al. [9] used a discrete element numerical modelling program, DECICE, to calculate interaction forces between ship and pack ice pieces. Wang et al. [10] developed a collision model for nonlinear dynamic finite element analysis on a LNG ship and a crushable ice using commercial code DYTRAN. Liu et al. [11] conducted nonlinear finite-element analysis by using a commercial code LS-DYNA to assess the internal mechanics of both icebergs and ship body. A plastic model was set for icebergs, where an empirical criterion was used to detect the failed ice element. Shi et al. [12] applied an elastic-perfect-plastic materiel model to simulate the collision between a FPSO ship and an iceberg. The gradient temperature effect was considered, where "Tsai-Wu" yield surface was applied in the simulations.

The interaction between the ice cusps fragmented from intact ice and the ship hull should not be ignored, as it affects both the ice failure patterns and ice resistance on the structure. The traditional FEM is based on the assumption of continuum material, and it is difficult to simulate the crack branching and the formation of the rubble accumulation. Paavilainen et al. $[13,14]$ combined FEM and DEM to simulate the interaction of an inclined plate with ice sheet. FEM was used to simulate continuous failure of ice sheet while DEM was used to simulate ice rubble pile. The model presents great advantages in modelling the ice accumulating process and bulking behaviour of force chains, and provides good idea for the numerical investigations on the ice-structure interaction. In this paper, a 3D numerical model is established by combining cohesive element method (CEM) with an elastoplastic softening constitutive model to simulate continuous icebreaking process in level ice. The local crushing of ice is presented by the elastoplastic softening constitutive model of ice material, while the cracks caused by bending failures are realized by cohesive element method. Firstly, the proposed numerical models are introduced and the ice elastoplastic constitutive model is calibrated through simulating an ice crushing experiment. Then the continuous icebreaking process is simulated by combining the elastoplastic constitutive model and cohesive element method. The mesh dependency study is carried out and the simulated ice resistance and ice failure patterns are compared with model test results. Finally, the effects of the ship velocity on ice resistance and ice failure patterns are investigated as well. 


\section{Numerical models}

\subsection{Elastoplastic softening model}

When a marine structure interacts with ice sheet, local crushing happens around the contact region. However, the mesh applied in the FEM simulation is always too coarse to present the feature of local crushing, i.e., such microscopic failure is difficult to be explicitly modeled in the simulation. Hilding et al. [15] proposed a numerical approach which took local crushing as a product of multiple micro-crack branching in the contact zone. An elastoplastic linear softening constitutive model, taken as a homogenization approach, was applied in the numerical model to present the influence of the arising and propagation of micro-cracks on ice material performance. The corresponding hardening curve is shown in Fig. 1.
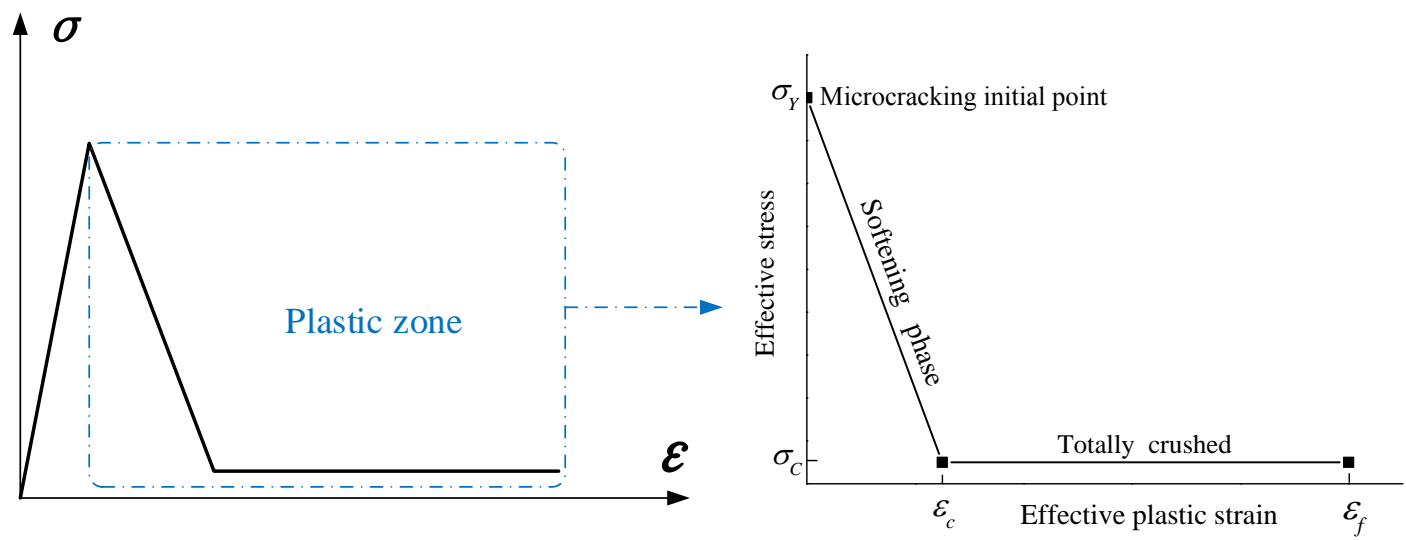

Fig. 1. Elastoplastic constitutive model given by Hilding et al. [15]

Before reaching the crushing initial point (yield stress $\sigma_{y}$ ), the ice material is elastic. After reaching the crushing initial point, ice material starts to undergo a linear softening process. The macroscopic effect of local crushing is that ice is becoming softened with the development of micro-cracks whose amount is proportional to the deformation. When the plastic strain reaches crushed plastic strain $\varepsilon_{c}$, ice element is totally crushed and it behaves as a viscous fluid. The corresponding effective stress at this point is crushed effective stress $\sigma_{c}$, whose value should be relatively low which means ice material cannot bear external load at this stage. Finally, a failure strain $\varepsilon_{f}$ is set to avoid the ice elements to be excessively distorted. It should be noted that the linear behavior during the softening phase was a rough assumption by Hilding et al. [15]. The diverse softening phases could be used to represent different stress-strain relationships.

\subsection{Cohesive element method}

Cohesive element method (CEM) is an extension of cohesive zone model (CZM) in FEM simulations. CZM was firstly proposed by Hillerborg et al. [16] to model the local crack propagation of an unreinforced concrete beam. In their study, the fracture around the crack tip was simulated and the behavior of cohesive elements was controlled by a traction-separation law. CZM was applied to analyzing the behavior of ice by Mulmule and Dempsey $[17,18]$. Afterwards, some other researchers [19-23] applied CEM to simulate the interactions between level ice and marine structures.

In the framework of CEM, the cohesive elements are inserted into every internal face between the neighboring finite bulk elements, as shown in Fig. 2. Theoretically, the cohesive 
element is a zero-thickness element which would respond to tension and shear on the cohesive surface. In practical simulation, the cohesive elements are defined as ultrathin elements for the numerical accuracy. The bulk elements and the cohesive elements share nodes, thus deformation and stress could transmit directly between them. When the separation of cohesive element reaches the critical value, the cohesive element is considered to be completely damaged and is deleted from the simulation. Consequently, the explicit crack is created along the cohesive element faces.

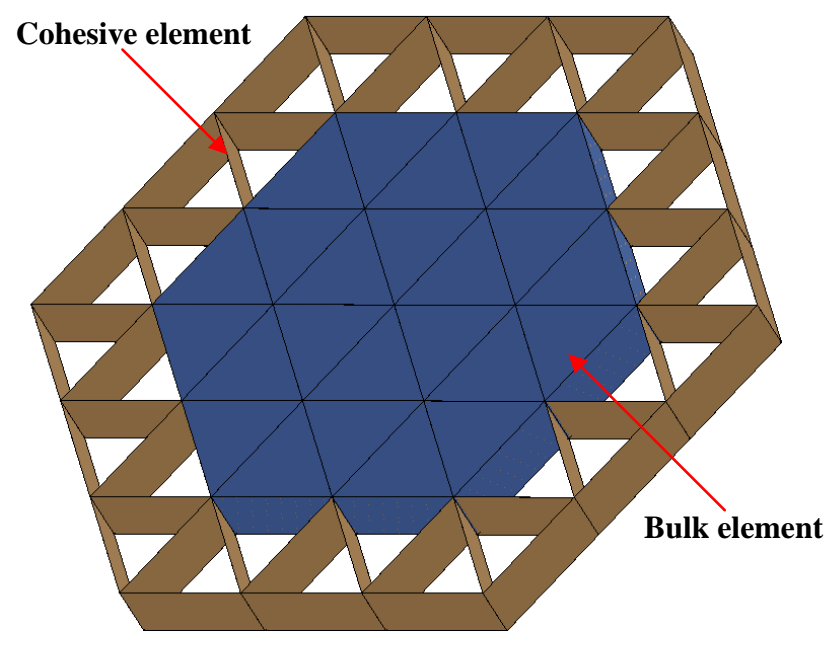

Fig. 2. Illustration of ice bulk elements and cohesive elements

Traditionally, 8-node three dimensional brick mesh, i.e., hexahedral mesh, is applied to bulk element for its good numerical property. However, the usage of hexahedral mesh will lead to a "zig-zag" crack path, which is longer than the actual path (e.g., $\sqrt{2}$ times for a $45^{\circ}$ crack). This will cause an extra dissipation of fracture energy and produce a non-negligible influence on the simulation results. In this paper, the 6-node regular tri-prism mesh as shown in Fig. 2 is applied to the bulk element to attenuate this effect. This kind of mesh has shown great advantages in modeling bending fracture and random propagation path of crack during the ice-conical structure interaction in the numerical research by Wang et al. [22].

The behaviors of cohesive elements are mainly controlled by three factors: fracture energy, fracture stress and traction-separation law (TSL) curve. Fracture energy is the dissipated energy by the failure of the cohesive elements and fracture stress is the maximum stress obtained in this process. The TSL curve represents the relation between the crack separation and the cohesive force, which can be seen as the constitutive law of cohesive elements. Fracture energy can be obtained by fracture mechanic tests. Based on the in-situ tests carried out by Dempsey et al. [24], the size-independent fracture energy of multi-year ice was within the range of $23 \sim 47 \mathrm{~J} / \mathrm{m}^{2}$. Normally, the fracture stresses are substituted by tensile strength in mode I fracture and shear strength in mode II fracture. Based on the test data from previous work, Timco and Weeks [25] obtained the tensile strength ranged from 0.2 MPa to $0.8 \mathrm{MPa}$, while the shear strength was within 0.55 0.9 MPa. According to the research by Cornec et al. [26] and Wang et al. [22], the shape of TSL curve brings limit effects on simulation results as long as the value of fracture energy is correct. The most commonly used traction-separation law in CEM is bilinear law, whose detailed expressions could refer to Wang et al. [23]. 


\section{Numerical calibration of the constitutive model}

Hilding et al. [15] roughly assumed the effective stress and plastic strain obey a linear softening relationship in the elastoplastic softening constitutive model. However, there are various softening laws could be applied and the effects of different shapes of softening laws should be evaluated. Fig. 3 shows three kinds of softening laws presented in this paper, i.e., linear law, exponential law and stepped law, which defines different ice softening process with the development of micro-cracks. A numerical study on ice cone crushing tests is performed to evaluate the effects of different softening laws on the simulation results, and also calibrates the proposed constitutive model in modeling ice local crushing failure. It should be noted that the cohesive element method is not introduced in this section.

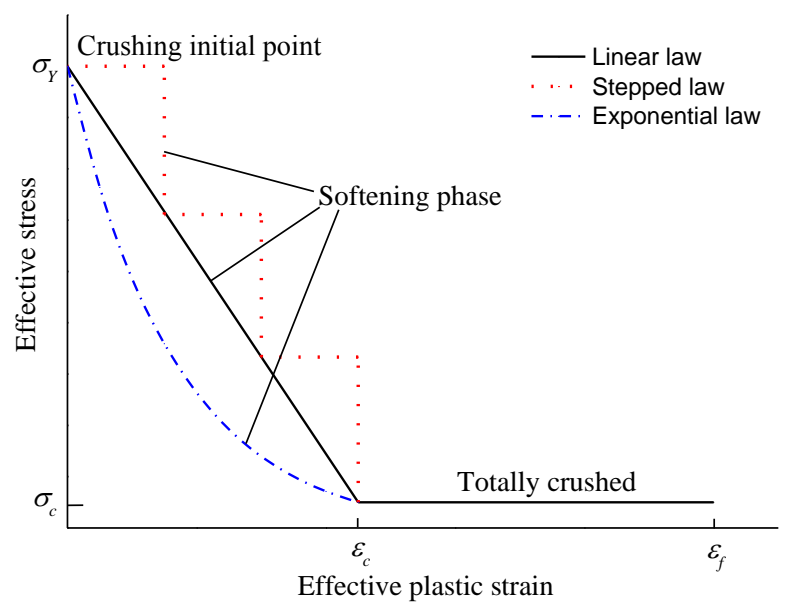

Fig. 3. Three kinds of softening laws applied in this paper

The numerical setup is based on the ice cone crushing tests carried out by Kim et al. [27]. As shown in Fig. 4, a steel indenter impacts on an ice cone with constant velocities. The diameter of ice cone is $D=250 \mathrm{~mm}$, and the inclined angle is $30^{\circ}$. The width and the length of the steel plate are $400 \mathrm{~mm} \times 400 \mathrm{~mm}$. The bottom of ice cone is fixed and a velocity boundary is set on the rigid plate by the command of Prescribed-Motion to keep the plate impacting against the ice cone with two constant velocities, $1 \mathrm{~mm} / \mathrm{s}$ and $100 \mathrm{~mm} / \mathrm{s}$. Based on the experimental data, the material parameters of ice cone are set as listed in Table 1. Three softening laws shown in Fig. 3 are applied in the ice elastoplastic constitutive model. The mesh size of ice cone is set as $l_{m}=2.5 \mathrm{~mm}$, which is determined by a prior mesh dependency study to obtain the convergent results. The material of the steel indenter is set as rigid without consideration of deformation, whose parameters are also shown in Table 1. Because the stiffness of ice cone is greatly different from that of rigid plate, the contact algorithm between ice cone and rigid plate adopts command of CONTACT-ERODING-NODES-TO-SURFACE, and the friction coefficient between ice and rigid plate is set as 0.2.

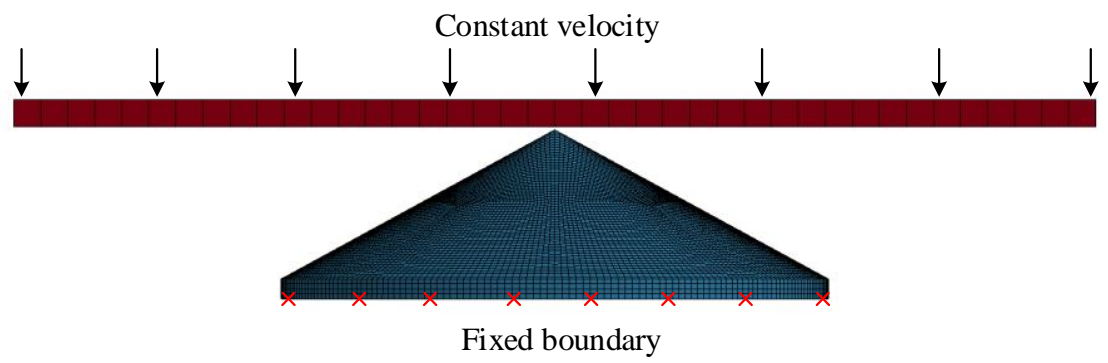

Fig. 4. Model of ice cone crushing against the rigid indenter 
Feng Wang, Li Zhou, Zao-Jian Zou

Ming Song, Yang Wang, Yi Liu
Study of continuous icebreaking process

with cohesive element method

Table 1 Material parameters of ice cone and rigid plate

\begin{tabular}{|l|c|l|c|}
\hline Ice cone & Value & Items & Value \\
\hline Items & 910 & Density $\left(\mathrm{kg} / \mathrm{m}^{3}\right)$ & 7850 \\
\hline Density $\left(\mathrm{kg} / \mathrm{m}^{3}\right)$ & 5 & Elastic modulus $(\mathrm{GPa})$ & 200 \\
\hline Elastic modulus $(\mathrm{GPa})$ & 0.3 & Poisson ratio & 0.3 \\
\hline Poisson ratio & 15 & & \\
\hline Yield stress $\sigma_{Y}(\mathrm{MPa})$ & 0.1 & & \\
\hline Crushed stress $\sigma_{c}(\mathrm{MPa})$ & 0.4 & & \\
\hline Crushed strain $\varepsilon_{c}$ & 0.5 & & \\
\hline Failure strain $\varepsilon_{f}$ & & \\
\hline
\end{tabular}

Fig. 5 gives the comparison between the numerical results and the experimental results from Kim et al. [27] at different impact velocities. As shown in the figure, all of the load curves present "stepped" rising tendency due to the continuous impacting and local crushing between ice cone and rigid plate. The gentle load drops appeared in the simulations are caused by the softening and failure of ice elements, which also can be observed in the experimental results. The abnormal large drops of experimental loads at the displacement of $9 \mathrm{~mm}$ and $18 \mathrm{~mm}$ are caused by start-stop discontinuity of the indenter motion, which was explained by Kim et al. [27]. The mean values and standard deviations of calculated and measured impact forces are given by Table 2, in which the errors between the calculated and measure results are also provided. Among the three softening laws, the linear law obtains the closest results to the experimental results, while the stepped law derives higher loads and exponential law derives lower loads. In addition, the load fluctuations with stepped law are obviously heavier than those with other two laws. This result is more obvious under high impact velocity. This is because that the stress varies unevenly with the strain and even experiences sudden drops at some strain points, which ultimately induces larger fluctuations in the load curves.

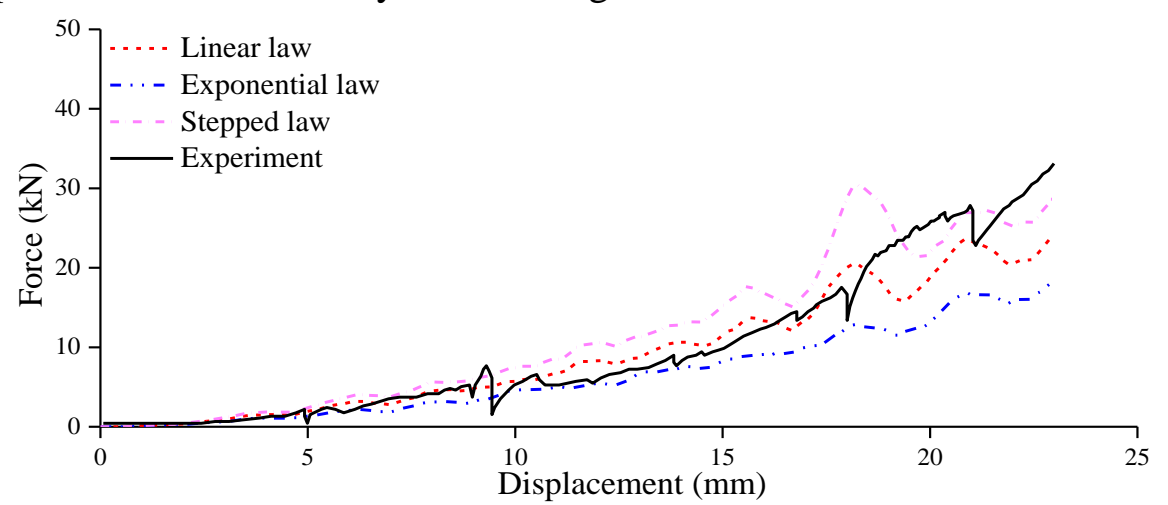

(a) $1 \mathrm{~mm} / \mathrm{s}$ impact velocity

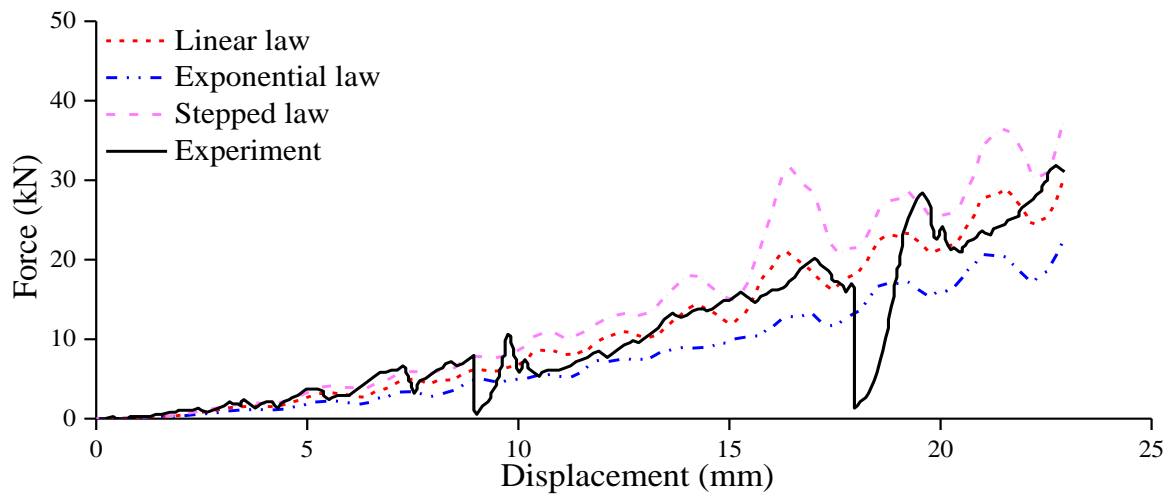

(b) $100 \mathrm{~mm} / \mathrm{s}$ impact velocity

Fig. 5. Comparison of impact forces between simulations and experiments 
Table 2 Comparison between simulated results and measured results

\begin{tabular}{|c|c|c|c|}
\hline Softening law & Impact velocity & Mean & StDev \\
\hline Linear law & \multirow{4}{*}{$1 \mathrm{~mm} / \mathrm{s}$} & $9.90 \mathrm{kN}(-7.5 \%)$ & $8.37 \mathrm{kN}(-5.1 \%)$ \\
\hline Exponential law & & $7.03 \mathrm{kN}(+34.3 \%)$ & $6.00 \mathrm{kN}(-32.0 \%)$ \\
\hline Stepped law & & $12.6 \mathrm{kN}(+17.8 \%)$ & $10.7 \mathrm{kN}(+21.3 \%)$ \\
\hline Model test & & $10.7 \mathrm{kN}(-)$ & $8.82 \mathrm{kN}(-)$ \\
\hline Linear law & \multirow{4}{*}{$100 \mathrm{~mm} / \mathrm{s}$} & $12.2 \mathrm{kN}(-0.81 \%)$ & $10.7 \mathrm{kN}(-6.14 \%)$ \\
\hline Exponential law & & $8.64 \mathrm{kN}(-29.8 \%)$ & $7.50 \mathrm{kN}(-34.2 \%)$ \\
\hline Stepped law & & $15.6 \mathrm{kN}(+26.8 \%)$ & $13.9 \mathrm{kN}(+21.9 \%)$ \\
\hline Model test & & $12.3 \mathrm{kN}(-)$ & $11.4(-)$ \\
\hline
\end{tabular}

Fig. 6 presents the results of the pressure-area relationship of the numerical and experimental results. The pressure is obtained by dividing impact force with nominal contact area, which is the contact area projected onto the rigid plate. It can be seen that the simulation results capture the same trend of the experimental results, i.e., the rapid decreasing trend of the pressure with the increase of nominal contact area. The general shapes of the scattered point distributions are close to the exponential form. The similar pressure-area relationship was also observed in the research by Masterson et al. [28] on the various ice-structure impacting model tests. The fluctuation of the pressure is most serious when the contact area is less than $1000 \mathrm{~mm} 2$. With the continuous increases of the contact area, the variation of the pressure tends to be gentle. The measured pressure arises abnormal sudden drops at area of $900 \mathrm{~mm}^{2}$ and $3000 \mathrm{~mm}^{2}$, which is caused by start-stop discontinuity of the indenter motion in the model tests. The fitted curves with the format of $y=a \cdot x^{b}$ for pressure-area relationship results are shown in Fig. 7. The simulation results with the linear law fit best with the experimental results, while the pressure obtained by stepped law is higher and the pressure obtained by exponential law is lower than the measured pressure.

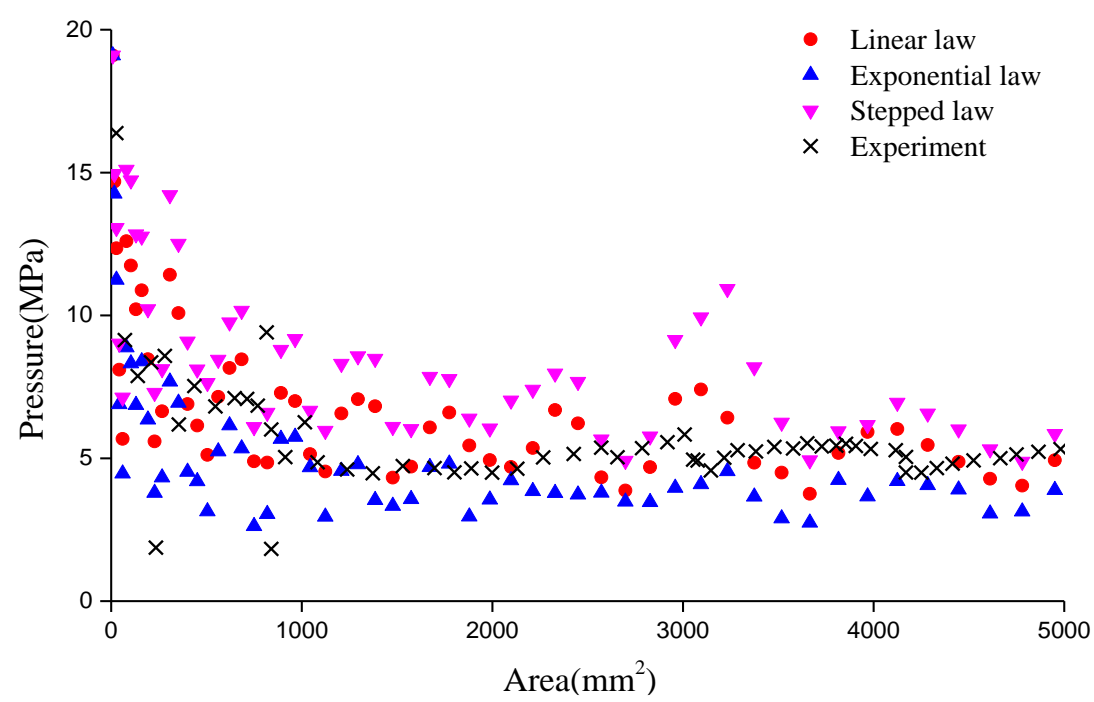

(a) impact velocity at $1 \mathrm{~mm} / \mathrm{s}$ 
Feng Wang, Li Zhou, Zao-Jian Zou Ming Song, Yang Wang, Yi Liu
Study of continuous icebreaking process with cohesive element method

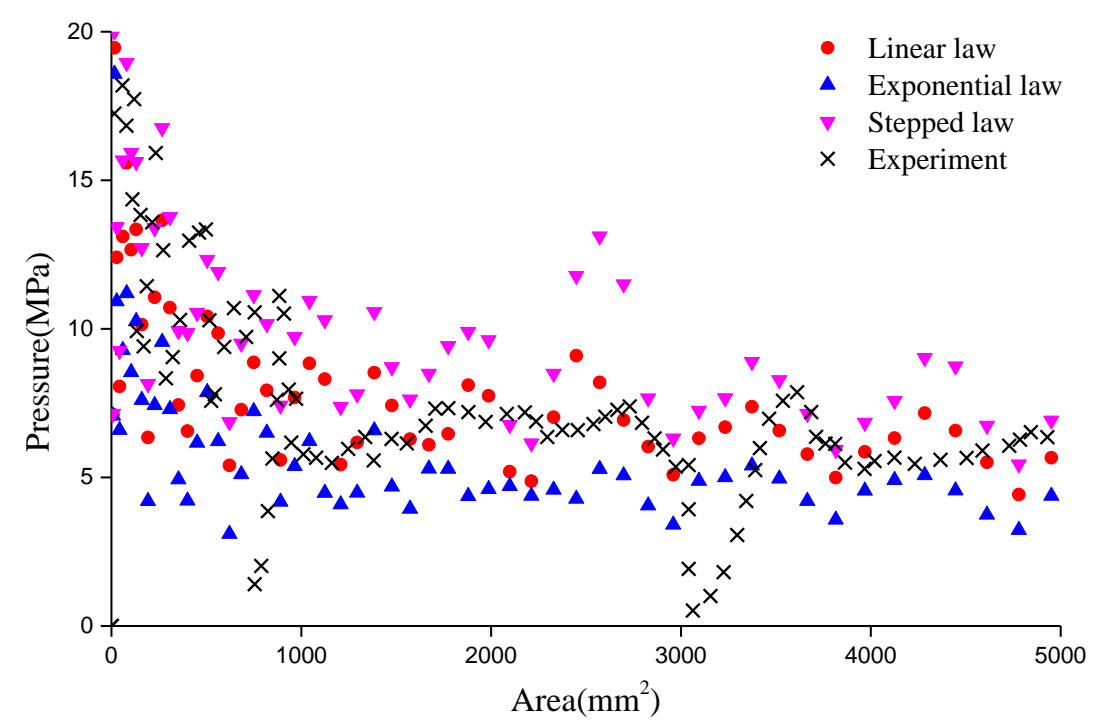

(b) impact velocity at $100 \mathrm{~mm} / \mathrm{s}$

Fig. 6. Comparison of pressure-area relationships between simulations and experiments

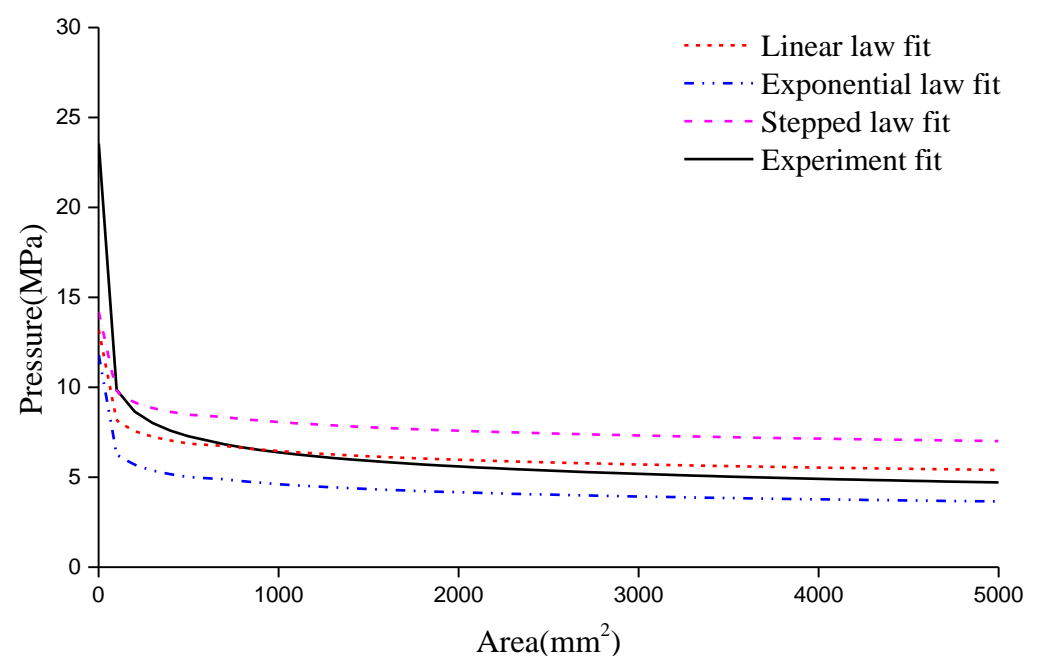

(a) impact velocity at $1 \mathrm{~mm} / \mathrm{s}$

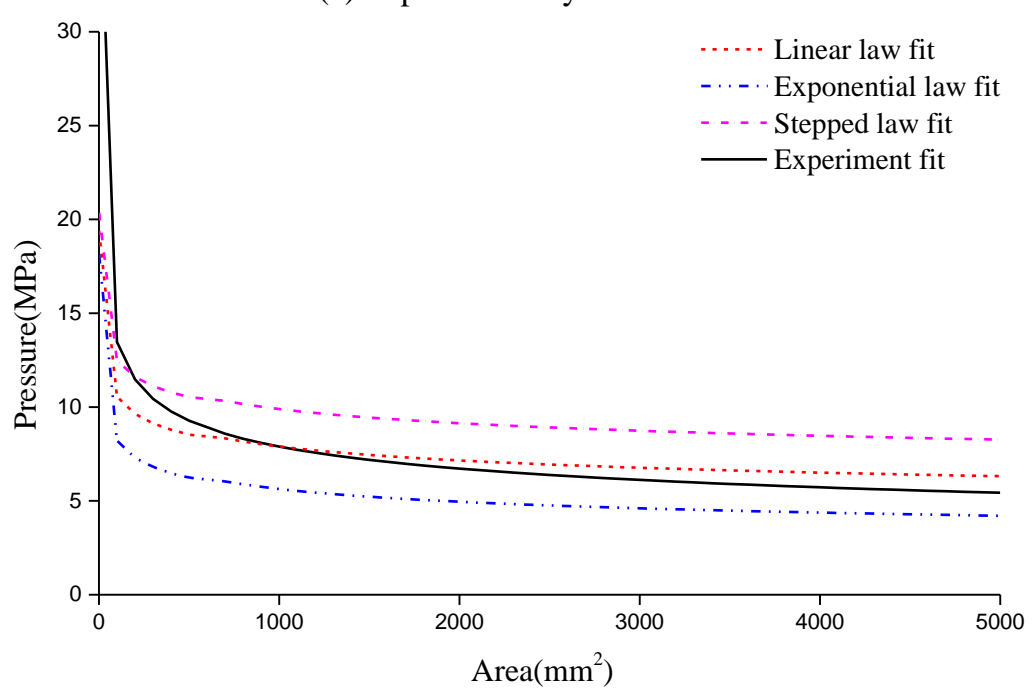

(b) impact velocity at $100 \mathrm{~mm} / \mathrm{s}$

Fig. 7. Comparison of fitted pressure-area curves between simulations and experiments 


\section{Simulation of continuous icebreaking process}

The numerical simulation of the continuous icebreaking process is presented in this section. The cohesive element method is introduced in this section to simulate the bending failure of level ice. In addition, the linear softening elastoplastic model, which obtains closest results to the experimental results, is chosen as the constitutive model of ice bulk element to simulate the local crushing of ice sheet.

\subsection{Geometry and numerical setup}

The double-hull icebreaking tanker, MT Uikku, is chosen as the object in the present study. Zhou et al. [29, 30] carried out a series of model tests in the multifunctional ice basin of the Marine Technology Group at Aalto University. The ship model was set to be even keel without heel angle, and the scale factor $\lambda=31.56$. The principal dimensions of the ship are listed in Table 3 and the profile of the hull is shown in Fig. 8. The parameters used in the simulations are in full scale based on Froude scaling.

Table 3 Principal dimensions of the icebreaking tanker - MT Uikku

\begin{tabular}{|l|c|c|}
\hline Items & Model scale & Full scale (simulation) \\
\hline Length $L(\mathrm{~m})$ & 4.75 & 150 \\
\hline Breadth $B(\mathrm{~m})$ & 0.67 & 21.3 \\
\hline Draft $T(\mathrm{~m})$ & 0.30 & 9.5 \\
\hline Block coefficient $C_{b}$ & 0.72 & 0.72 \\
\hline
\end{tabular}

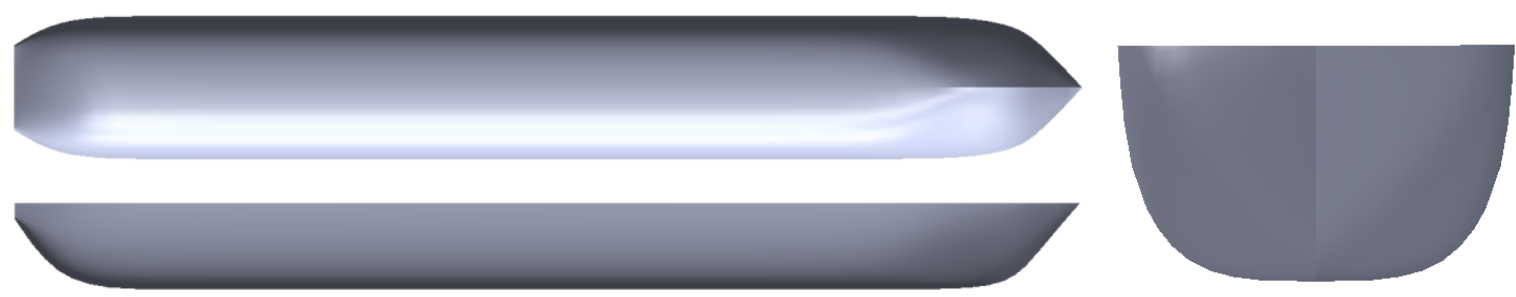

Fig. 8. Profile of the icebreaking tanker - MT Uikku

In the model tests, the ship was towed by a carriage to keep a constant velocity. The 6component force transducer and cameras were attached to the ship model to measure ice resistance and record the icebreaking process. Three drift angles $\left(0^{\circ}, 45^{\circ}\right.$ and $\left.90^{\circ}\right)$ were tested in the model test. Here, only the data from cases with drift angle $0^{\circ}$ are utilized to compare with numerical results. The ship velocities and measured ice properties in model scale and full scale are listed in Table 4. The numerical model of full-scaled continuous icebreaking scenario is established in LS-DYNA as shown in Fig. 9.

Table 4 Ship velocity and measured ice properties in model scale and full scale

\begin{tabular}{|c|c|c|c|c|c|c|c|c|}
\hline \multirow{2}{*}{ Case No. } & \multicolumn{2}{|c|}{$\begin{array}{c}\text { Ice thickness } \\
h(\mathrm{~mm})\end{array}$} & \multicolumn{2}{c|}{$\begin{array}{c}\text { Ship velocity } \\
V(\mathrm{~m} / \mathrm{s})\end{array}$} & \multicolumn{2}{c|}{$\begin{array}{c}\text { Elastic modulus } \\
E_{i}(\mathrm{MPa})\end{array}$} & \multicolumn{2}{c|}{$\begin{array}{c}\text { Compressive strength } \\
\sigma_{Y}(\mathrm{kPa})\end{array}$} \\
\cline { 2 - 9 } & Model & Full & Model & Full & Model & Full & Model & Full \\
\hline 103 & 24.5 & 770 & 0.0365 & 0.2 & 29 & 929 & 55 & 1748 \\
\hline 104 & 24 & 760 & 0.089 & 0.5 & 31 & 984 & 69 & 2192 \\
\hline 205 & 30.5 & 960 & 0.0365 & 0.2 & 53 & 1685 & 58 & 1840 \\
\hline 206 & 30 & 950 & 0.089 & 0.5 & 54 & 1701 & 59 & 1862 \\
\hline
\end{tabular}


The velocity boundary condition is imposed on the ship by the command of PrescribedMotion to ensure that the ship impacts with level ice with the constant velocity. The ship is free to move in the $x$ direction while the motions in other directions are constrained. This is in accordance with the setup in the model tests. The length of ice sheet is two times of ship length and the width is 4.5 times of ship breadth, which can reduce the computational burden and also help to eliminate boundary effect. The boundary condition of the three sides of ice sheet without contacting with ship is set as fixed to represent the restraining effect of the boundless level ice around the established ice domain. Meanwhile, non-reflecting boundary conditions are also set on these boundaries to further eliminate boundary effect. The mesh of ship and ice sheet are shown in Fig. 10. The ice elements are constituted by regular tri-prism bulk elements and ultrathin hexahedral cohesive elements, whose layout can refer to Fig. 2. There is only one layer of ice elements in the vertical direction and the thickness of ice cohesive elements is defined to be $1 \%$ of the length of ice bulk elements. Besides the parameters measured in the model tests as listed in Table 4, the other parameters of ice bulk elements and cohesive elements are given in Table 5. The material of ship is set as rigid steel without consideration of deformation during the interaction.

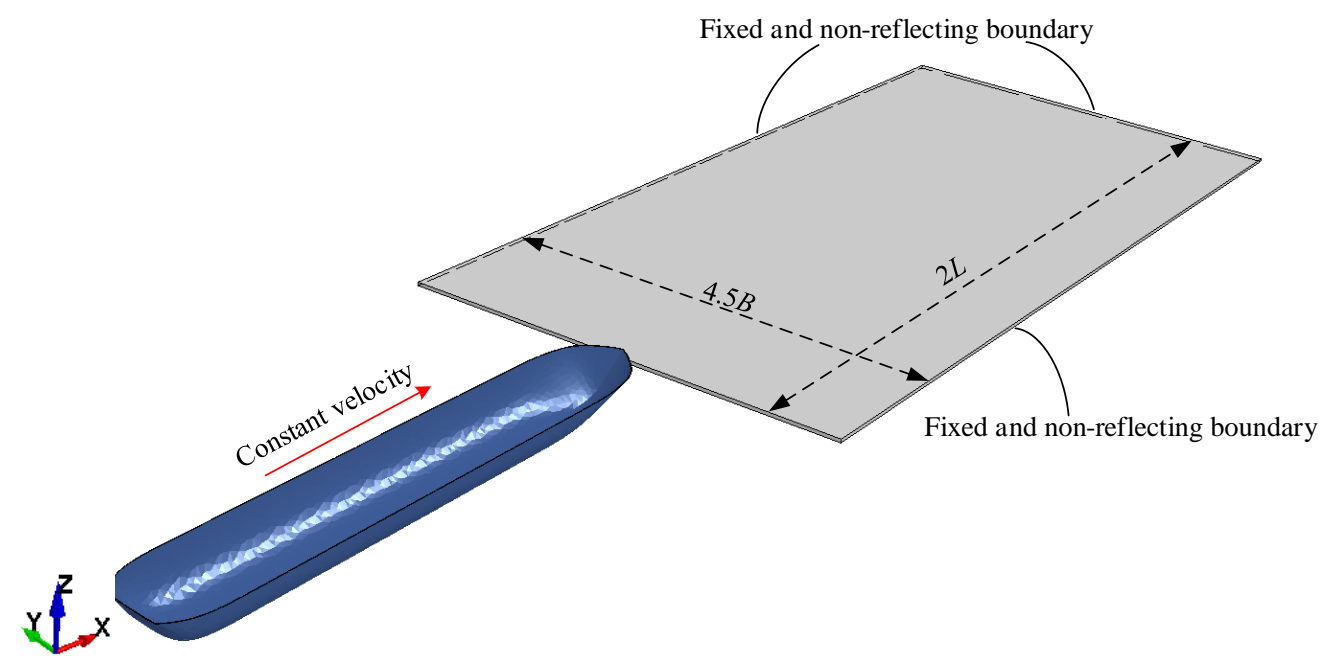

Fig. 9. Numerical model of continuous icebreaking scenario

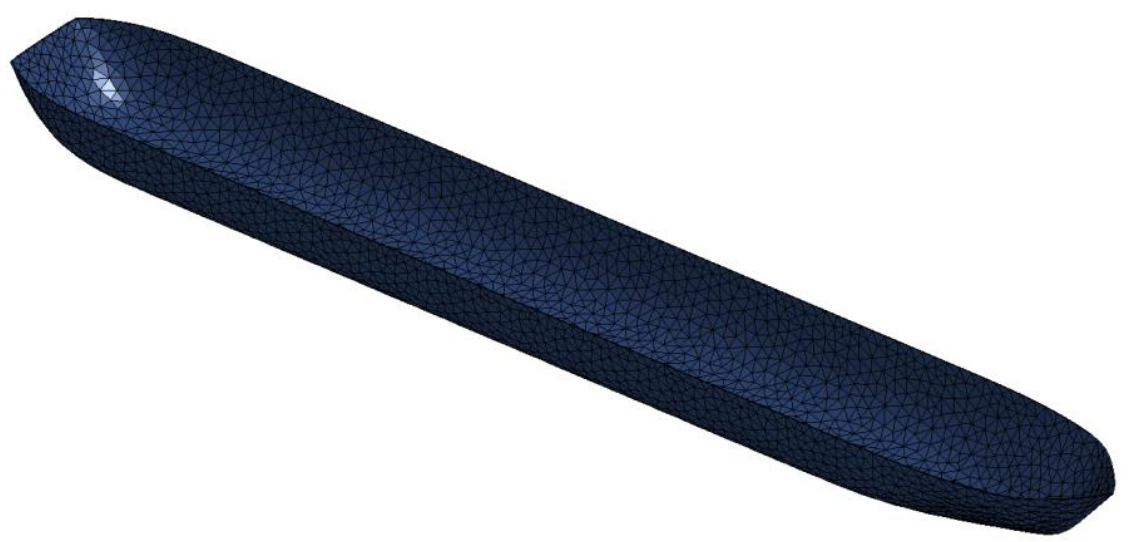

(a) Ship mesh 


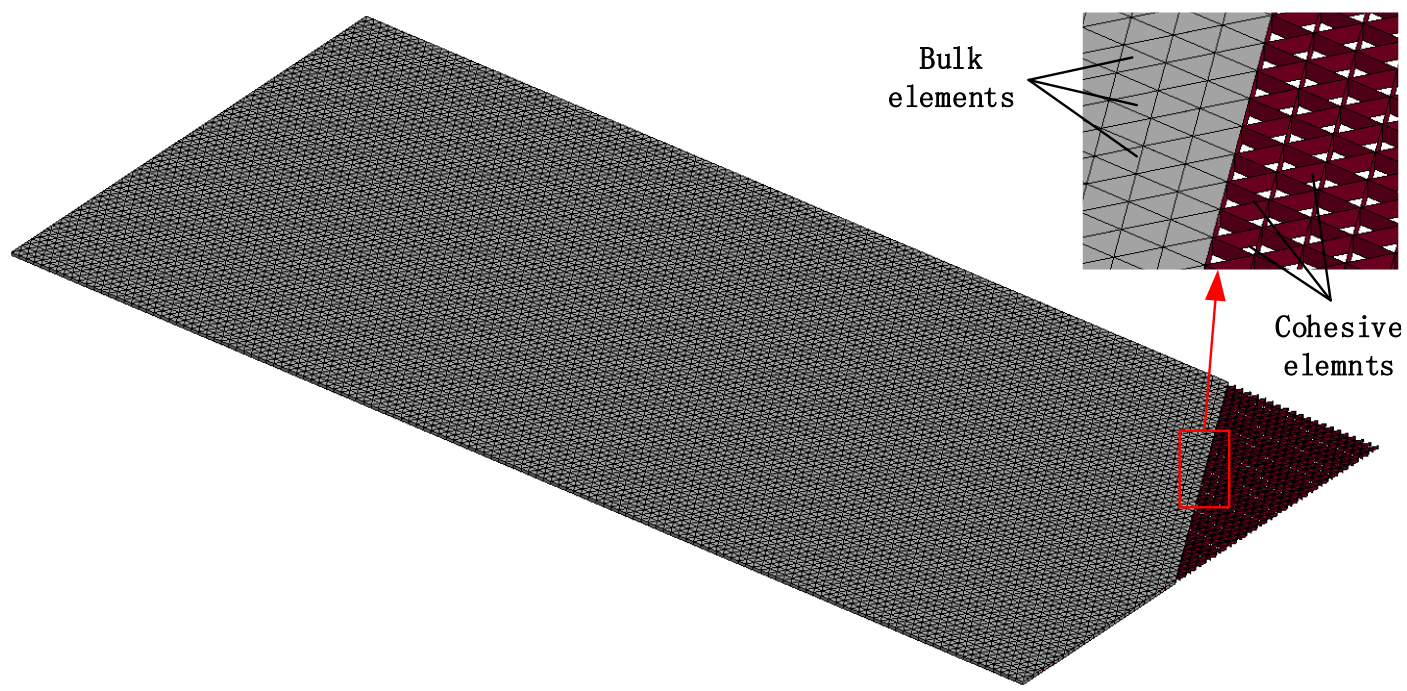

(b) Ice sheet mesh

Fig. 10 The mesh of ship and ice sheet

Table 5 Material parameters of ice bulk elements and cohesive elements

\begin{tabular}{|c|c|c|c|}
\hline \multicolumn{2}{|l|}{ Bulk elements } & \multicolumn{2}{|l|}{ Cohesive elements } \\
\hline Items & Value & Items & Value \\
\hline Density $\rho_{i}\left(\mathrm{~kg} / \mathrm{m}^{3}\right)$ & 910 & Density $\rho_{i}\left(\mathrm{~kg} / \mathrm{m}^{3}\right)$ & 910 \\
\hline Elastic modulus $E_{i}(\mathrm{MPa})$ & see Table 4 & Tensile strength $\sigma_{T}(\mathrm{kPa})$ & 500 \\
\hline Poisson ratio $v$ & 0.3 & Shear strength $\sigma_{S}(\mathrm{kPa})$ & 700 \\
\hline Compressive stress $\sigma_{Y}(\mathrm{kPa})$ & see Table 4 & Fracture energy in mode I $G_{I C}\left(\mathrm{~J} / \mathrm{m}^{2}\right)$ & 30 \\
\hline Crushed stress $\sigma_{c}(\mathrm{kPa})$ & 100 & Fracture energy in mode II $G_{I I C}\left(\mathrm{~J} / \mathrm{m}^{2}\right)$ & 30 \\
\hline Crushed strain $\varepsilon_{c}$ & 0.4 & Shape of TSL curve & Bilineal \\
\hline Failure strain $\varepsilon_{f}$ & 0.5 & & \\
\hline
\end{tabular}

In LS-DYNA, there are various of contact algorithms to treat the contact problems between the structures with different stiffness. The command of CONTACT-ERODINGNODES-TO-SURFACE is applied to treat interaction between ice and ship, and the command of CONTACT-ERODING-SINGLE-SURFACE is applied to treat interaction between ice and ice. Friction coefficient of 0.2 is set to the contact between ice and ship, while friction coefficient of 0.1 is set to the contact between ice and ice. In addition, the simplified buoyancy and damping models are established to simulate the interaction between ice and water base and incorporated in LS-DYNA solver by a user-defined subroutine. More details could be found in Wang et al. [22].

\subsection{Mesh dependency study and validation of numerical methods}

The mesh dependency study and validation of numerical methods are performed by comparing with experimental results in this section. In the previous numerical investigations on cohesive element method [21,22], it was found that the mesh size had an effect on the simulation results. In order to examine if such effect exists in the present numerical simulations, the mesh dependency study is carried out firstly. Based on the icebreaking scenarios as shown in Table 4, the ice resistance during the ship moving one hull length in ice sheet is calculated with three kinds of ice meshes, whose horizontal length are $4 \mathrm{~m}, 2 \mathrm{~m}$ and $1 \mathrm{~m}$. Label them as coarse mesh, medium mesh and dense mesh respectively.

Fig. 11 shows the relative proportion of mean resistance calculated with three meshes to those of model tests (the resistance obtained from the model tests has been converted to full- 
scale values based on Froude criterion). It can be seen that the mean forces present growing and convergent trends with the refinement of mesh. Compared to the experimental results, the medium mesh obtains the closest results with the error of $-8 \% \sim 7 \%$, while dense mesh slightly overestimates the forces for $7 \% \sim 18 \%$ and coarse mesh obtains obviously lower forces with the relative proportion of $60 \% \sim 77 \%$.

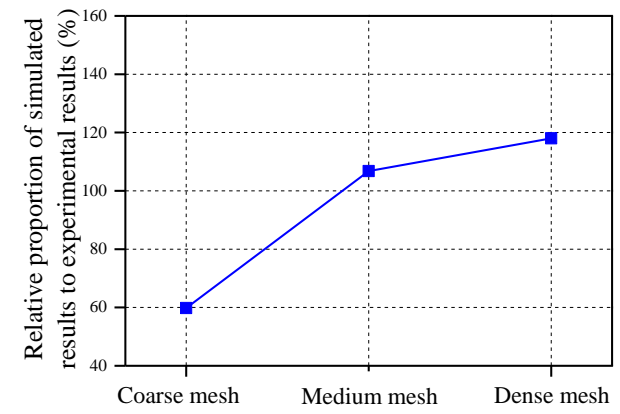

(a) Case 103

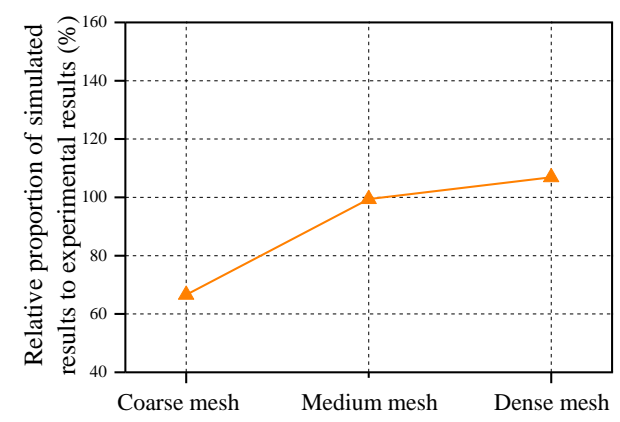

(c) Case 205

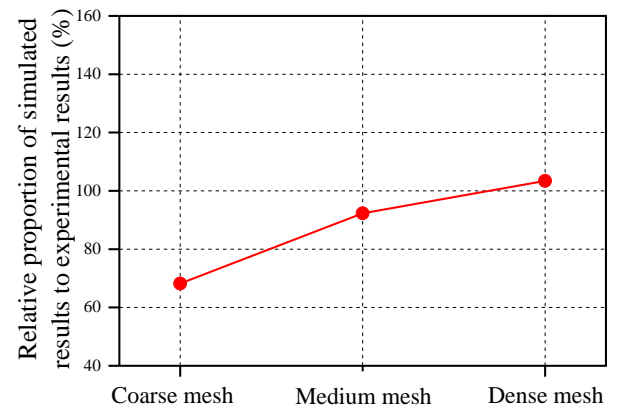

(b) Case 104

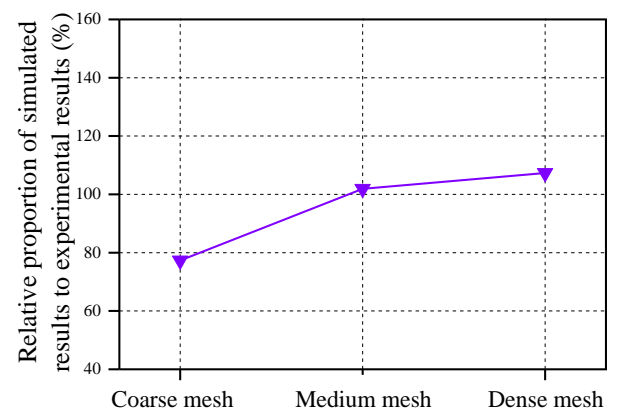

(d) Case 206

Fig. 11 Relative proportion of simulated mean ice resistance with different meshes to experimental results

$\mathrm{Lu}$ et al. [21] provided an explanation for this result. He found that the typical ice breaking length in the radial direction is about $2 \sim 3$ times of the ice thickness based on the observations in the model tests of the ice-conical structure interaction. With a mesh size similar to the actual ice breaking length in the radial direction can help to approximate the size of crack and obtain a better simulation result. Based on the measurements in the model tests by Zhou et al. [29], the full-scaled radial ice breaking length during icebreaking process varies within $1.58 \mathrm{~m} \sim 3.16 \mathrm{~m}$, whose mean value is rather close to the horizontal length of medium mesh applied in the present simulations. The simulation results provide another evidence for the conclusion by Lu et al. [21]. Thus, taking the advantage of the length being close to ice radial breaking length, the medium mesh is applied in the following simulations for the accurate prediction of ice resistance. Table 6 gives the mean values and standard deviations of ice resistance from experiments and simulations with medium mesh.

Table 6 Mean and standard deviations of ice resistance for experiments and simulations with medium mesh

\begin{tabular}{|c|c|c|c|c|}
\hline \multirow{2}{*}{ Case No. } & \multicolumn{2}{|c|}{ Model tests } & \multicolumn{2}{c|}{ Simulations } \\
\cline { 2 - 5 } & Mean $(\mathrm{kN})$ & StDev $(\mathrm{kN})$ & Mean $(\mathrm{kN})$ & StDev $(\mathrm{kN})$ \\
\hline 103 & 311 & 170 & 332 & 230 \\
\hline 104 & 415 & 185 & 383 & 272 \\
\hline 205 & 525 & 217 & 522 & 288 \\
\hline 206 & 617 & 333 & 629 & 410 \\
\hline
\end{tabular}

Fig. 12 shows the comparison of time histories of horizontal ice force between the model tests and the numerical simulations with medium mesh in the range of $50 \mathrm{~m}$ to $150 \mathrm{~m}$. In 
order to eliminate the noise from the results, the presented force curves are obtained by processing the original data through the same filter. It is observed that the numerical results agree well with the experimental results, in which both the loads curves show the considerable oscillation around the mean value. The fluctuation amplitudes of calculated ice resistance are slightly higher than those of measured loads, which will be explained later.

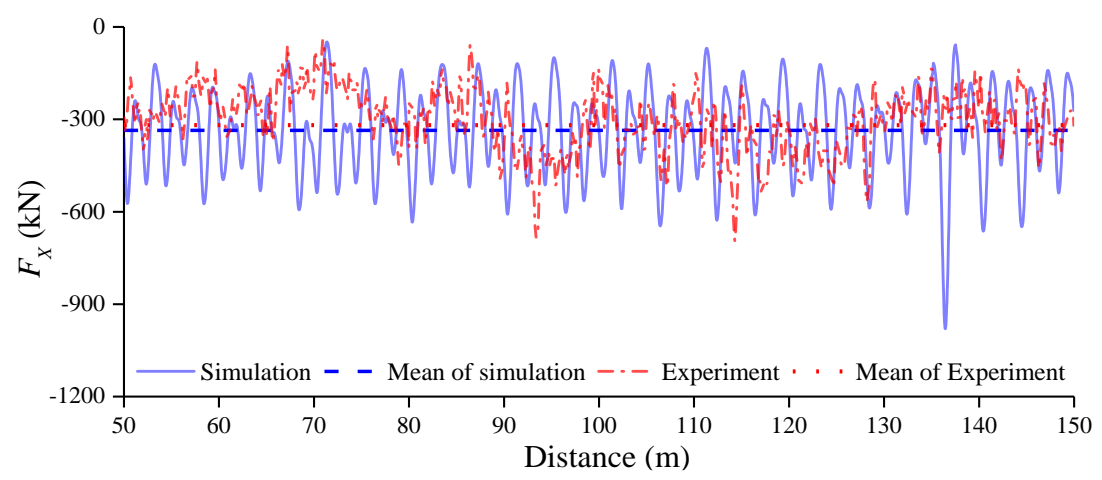

(a) Case 103

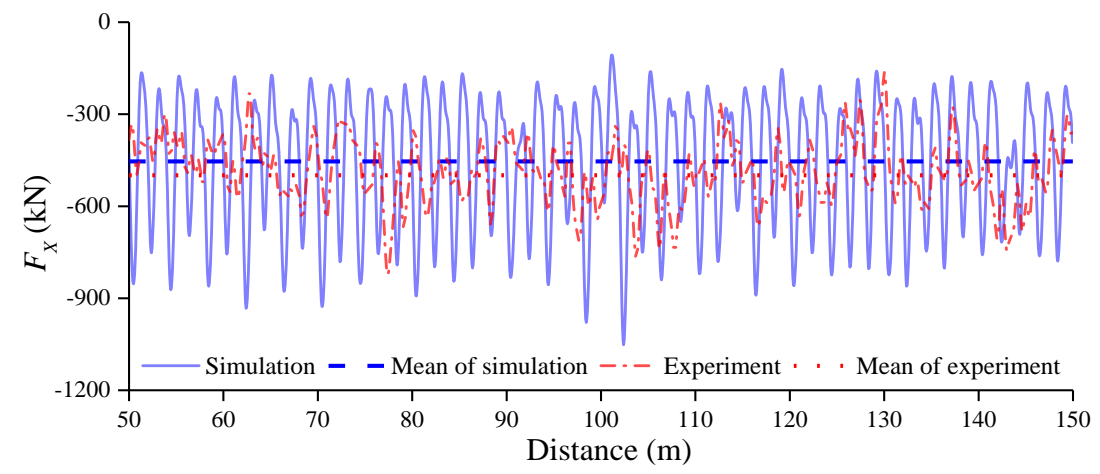

(b) Case 104

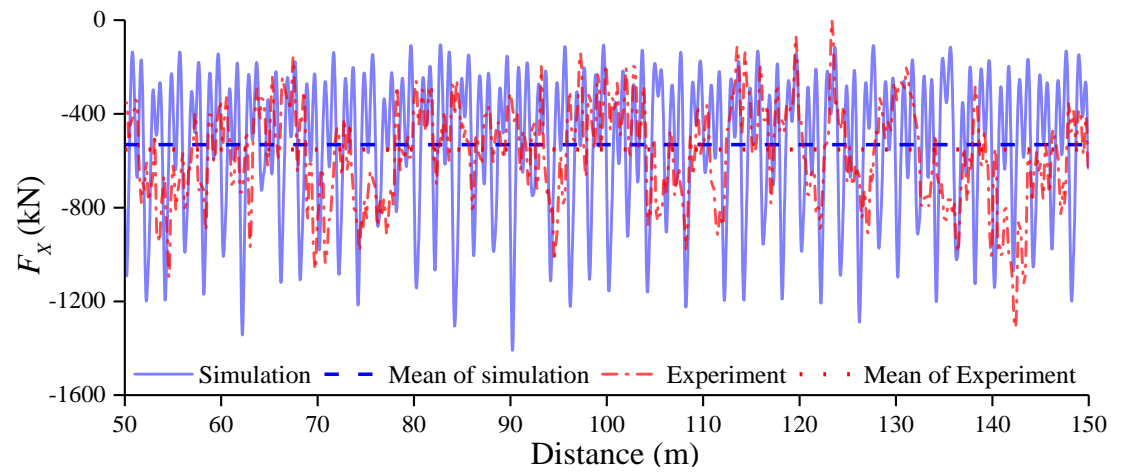

(c) Case 205

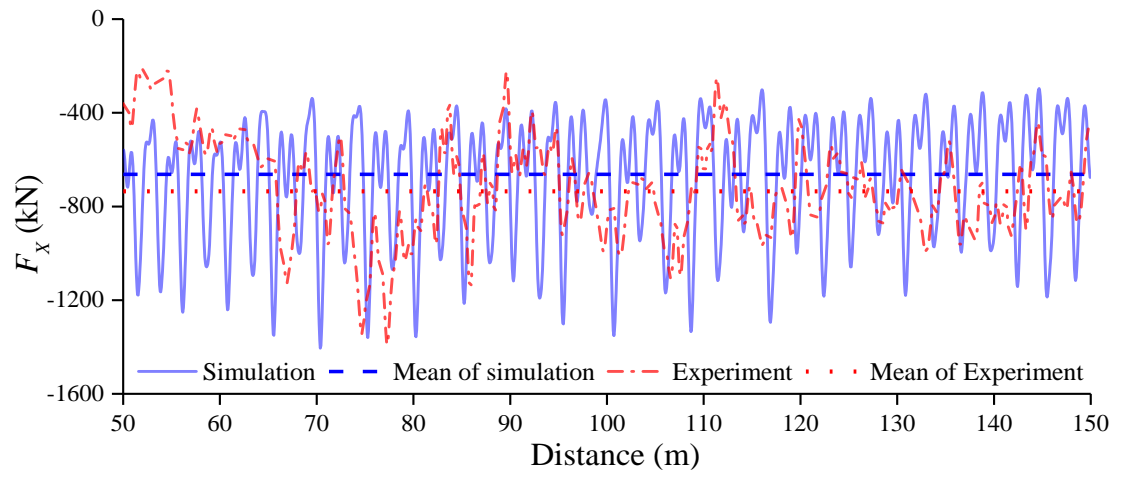

(d) Case 206

Fig. 12 Time histories of longitudinal force from experiments and simulations 
Fig. 13 shows the power spectra analysis of longitudinal ice forces on the hull. It is observed that both the simulated and measured results show one or two obvious spectral peaks. The frequencies corresponding to these peaks are the main ice-breaking frequency, which represents the impulse period of the ice bending-induced force on the hull. It can be observed that the ice bending-induced force is low frequency force, i.e., the spectral peaks appear under $2 \mathrm{~Hz}$. The spectral peaks of the model test appear around $0.2 \sim 0.6 \mathrm{~Hz}$, while the simulation results give a slightly higher frequency of $0.6 \sim 1.4 \mathrm{~Hz}$. In addition, there are some low-power energy distributions in both of simulated and experimental spectra. This is mainly caused by local crushing force and the contact force between ice cusps and hull (e.g., the accumulating force and friction force during the ice cusps sliding along the hull). These energy levels are obviously lower than the energy peaks due to ice bending-induced force, which indicates that the ice bending failure is the dominating ice failure pattern during the continuous icebreaking process.

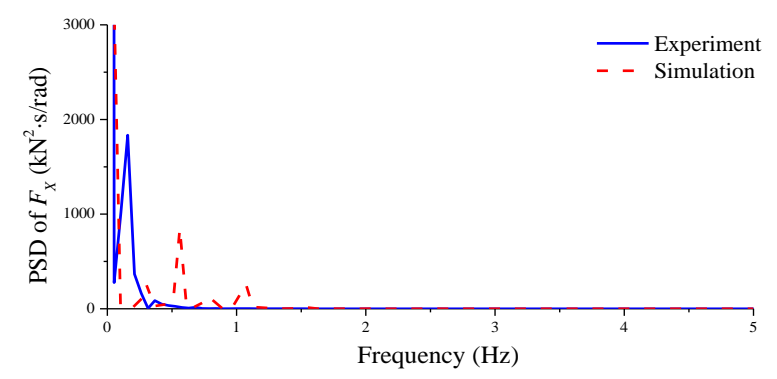

(a) Case 103

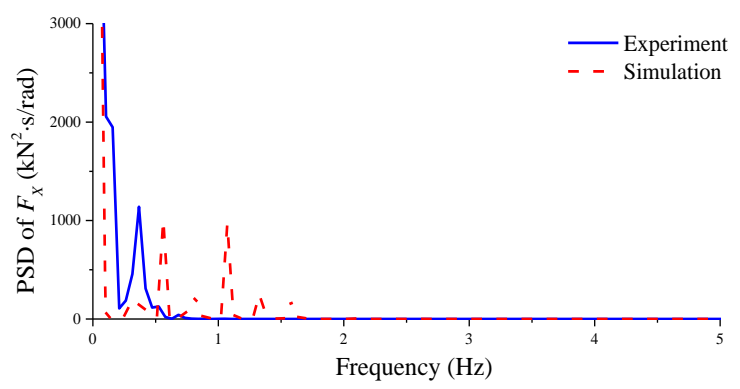

(c) Case 205

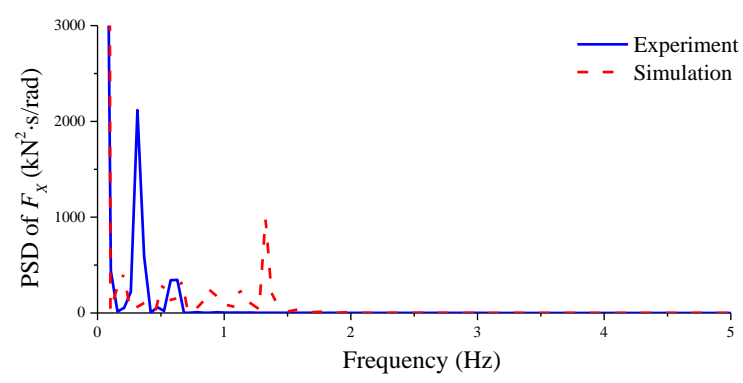

(b) Case 104

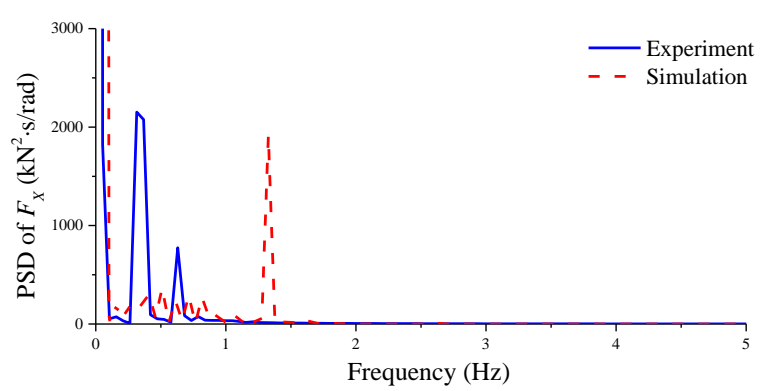

(d) Case 206

Fig. 13 Power spectra analysis of longitudinal ice forces on the hull

Taken Case 205 as an example, the simulated continuous icebreaking process are presented in Fig. 14. The outline of generated icebreaking channel is highlighted by the red lines in Figs. 14a c. In order to facilitate the observation of ice bending failure, the ice bulk elements are hided and only the ice cohesive elements are shown in Figs. 14d f. The whole continuous icebreaking process can be observed in the simulation results. At the initial stage of ship-ice interaction (see Fig. 14a, d), the ice local crushing happens at the contact zone. There is no obvious bending failure at this stage due to small contact area between bow and level ice. With the continuous advance of ship and the increase of contact area, the bending failure of ice sheet starts to arise. The initial crack appears at the ship shoulder zone and propagates towards outboard. The irregular icebreaking channel and large ice cusps are generated due to ice bending failure, which can be clearly observed in Fig. 14b c. The large ice cusps rotate and collide against the hull at the waterline zone under the action of buoyancy and gravity (see Fig. 14e $\mathbf{f}$ ). Fig. 15 shows the ice failure patterns observed in the model test for Case 205. The similar phenomenon of ice cusps rotation was also observed in the model test. From Fig.15c, it is shown that the shape of icebreaking channel in the model test is irregular, which is caused by the asymmetric and non-simultaneous ice failure patterns at the 
two sides of hull. A good agreement is achieved between simulated results and experimental results.

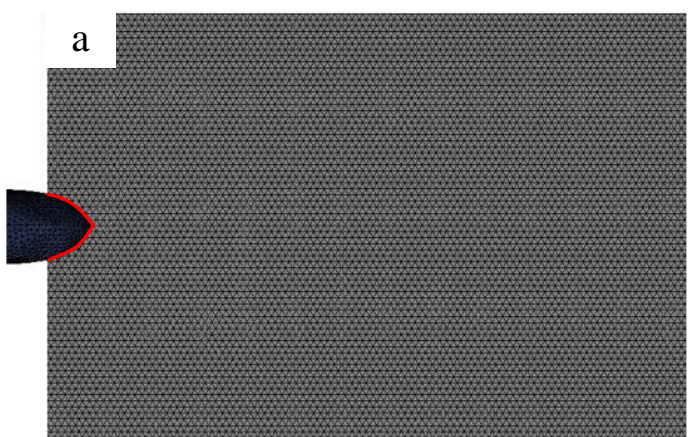

d
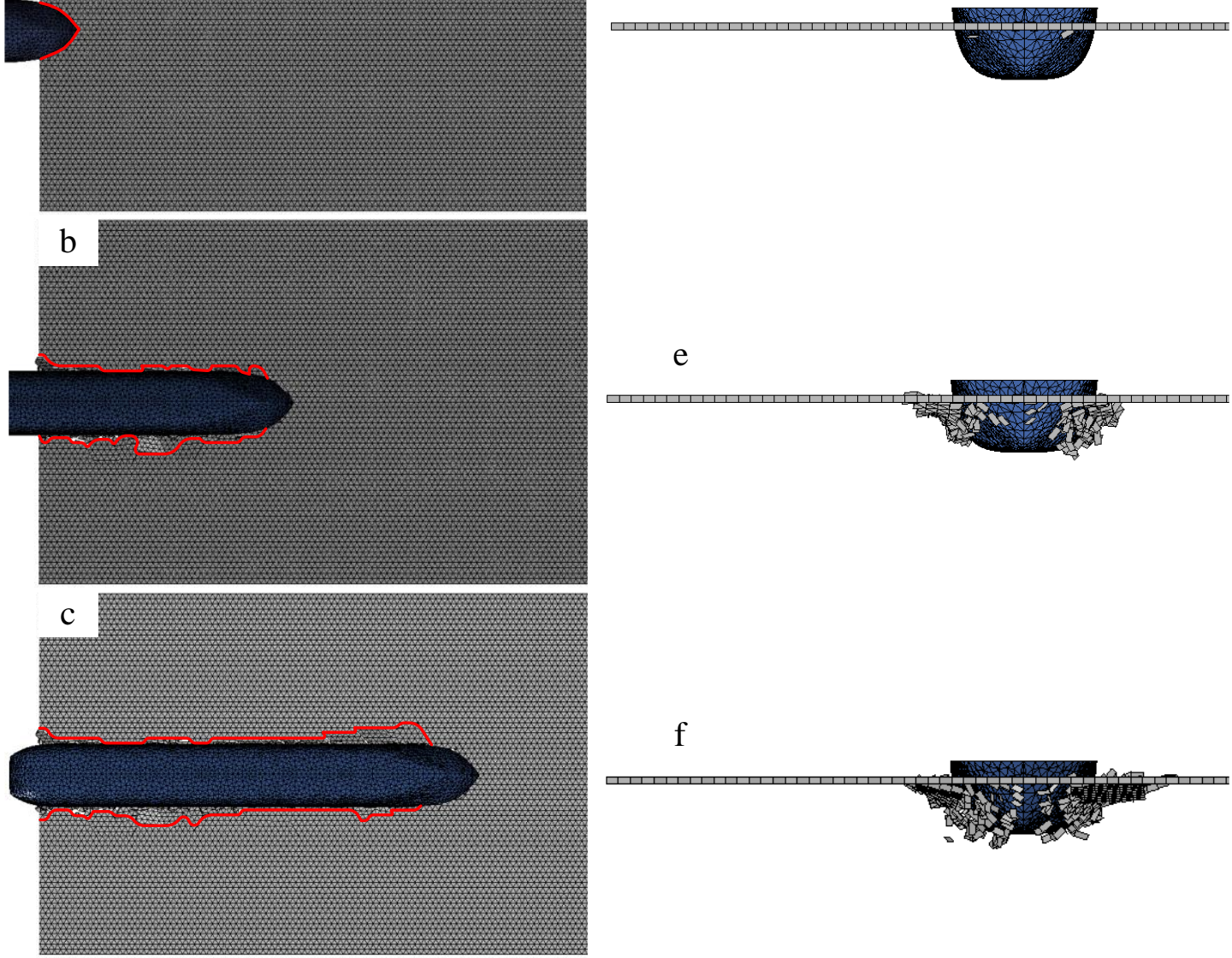

Fig. 14 Snapshots of simulated continuous icebreaking process for Case 205 (a. $t=50 \mathrm{~s}$, b. $t=350 \mathrm{~s}$, c. $t=700 \mathrm{~s}$, top view; d. $t=50 \mathrm{~s}$, e. $t=350 \mathrm{~s}, \mathrm{f} . t=700 \mathrm{~s}$, front view)
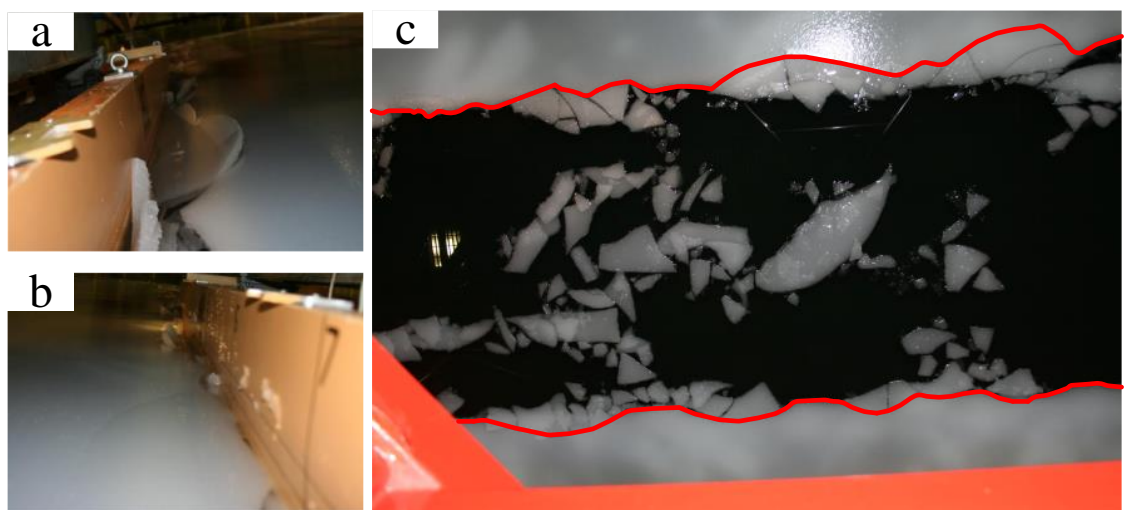

Fig. 15 Snapshots of the ice failure patterns in the model test for Case 205 (a. failure patterns at the starboard side; b. failure patterns at the port side; c. outline of icebreaking channel)

Fig. 16 shows the side view of simulated icebreaking process for Case 205 at $t=700$ s. It can be seen that the broken ice cusps slide backwards along the hull surface. There is not 
much rubble accumulation around the ship bow due to the smooth shape of bow. The ice accumulation mainly distributes at the both sides and bottom of hull. As the ship moves forward, ice cusps slide along the hull surface to the stern. During the ice sliding process, the friction will be imposed on the hull. From Fig. 16, it can also be seen the generated ice cusps with various sizes and shapes are rather similar to those observed in Fig. 15c. However, the width of large ice cusps generated in the numerical simulation are slightly larger than those observed in the model test, which indicates that the simulated ice breaking length is slightly larger than the experimental result. Therefore, when the ice sheet experiences bending failure in the numerical simulation, greater loads fluctuation will occur. This is the reason that the fluctuation amplitudes of ice resistance obtained in the numerical simulations are larger than the experimental results as shown in Fig. 12.

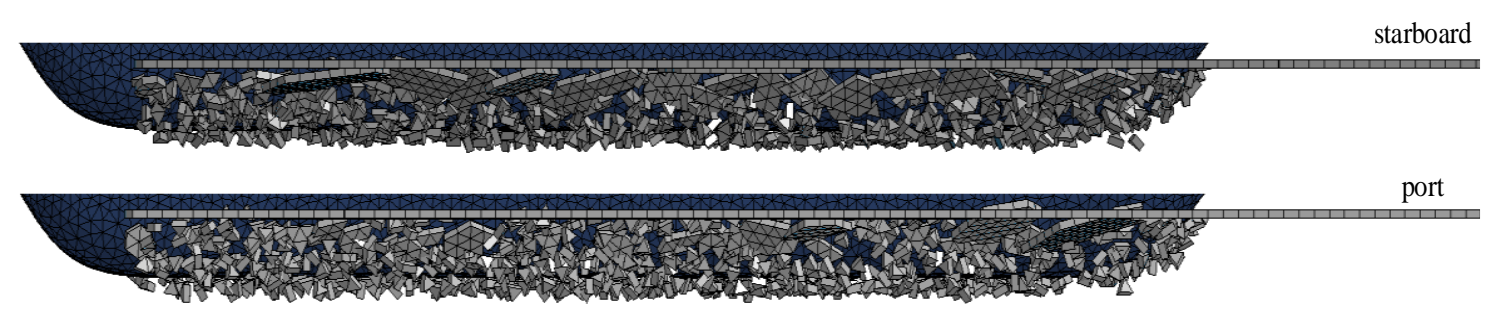

Fig. 16 Side view of simulated icebreaking patterns for Case 205 at $t=700 \mathrm{~s}$

\subsection{Effect of ship velocity}

The validation of the numerical method has been performed by comparing with the model test results in the last section. The influence of ship velocity $V$ on ice resistance and ice failure patterns will be studied in this section. Five ship velocities, $0.2 \mathrm{~m} / \mathrm{s}, 0.35 \mathrm{~m} / \mathrm{s}, 0.5 \mathrm{~m} / \mathrm{s}$, $0.65 \mathrm{~m} / \mathrm{s}$ and $0.8 \mathrm{~m} / \mathrm{s}$, are selected and the thickness of ice sheet are $760 \mathrm{~mm}$ and $950 \mathrm{~mm}$. Since the model tests only contain the scenarios with ship velocities of $0.2 \mathrm{~m} / \mathrm{s}$ and $0.5 \mathrm{~m} / \mathrm{s}$, the semiempirical formulas proposed by Lindqvist [5] and Riska et al. [7] are introduced for the comparison purpose.

The Lindqvist semi-empirical formulas are expressed as:

$$
\begin{aligned}
& R_{i}=\left(R_{c}+R_{b}\right)\left(\frac{1+1.4 V}{\sqrt{g h}}\right)+R_{s}\left(\frac{1+9.4 V}{\sqrt{g L}}\right) \\
& R_{c}=0.5 \sigma_{b} h^{2}\left(\tan \phi+\mu \frac{\cos \phi}{\cos \psi}\right) /\left(1-\mu \frac{\sin \phi}{\cos \psi}\right) \\
& R_{b}=\frac{27}{64} \sigma_{b} B \frac{h^{1.5}}{\sqrt{\frac{E}{12\left(1-v^{2}\right) g \rho_{w}}}}\left(\tan \psi+\mu \frac{\cos \phi}{\cos \psi \sin \alpha}\right)\left(1+\frac{1}{\cos \psi}\right) \\
& R_{s}=\left(\rho_{w}-\rho_{i}\right) g h B\left(T \frac{B+T}{B+2 T}+\mu\left(0.7 L-\frac{T}{\tan \phi}-\frac{B}{4 \tan \alpha}+T \cos \phi \cos \psi \sqrt{\frac{1}{\sin \phi^{2}}+\frac{1}{\tan \alpha^{2}}}\right)\right)
\end{aligned}
$$

where $R_{i}, R_{c}, R_{b}$, and $R_{s}$ are the total ice resistance, crushing resistance, bending resistance and submersion resistance, respectively. $\sigma_{b}$ is the ice bending strength, which are $828 \mathrm{kPa}$ for $760 \mathrm{~mm}$ thick ice and $920 \mathrm{kPa}$ for $950 \mathrm{~mm}$ thick ice, based on the measurements in the model tests by Zhou et al. [29]. $\mu$ is the friction coefficient between the ship and the ice, and $\rho_{w}$ is the water density. $\phi$ is the stem angle, $\alpha$ is the waterline entrance angle, and $\psi$ is the normal angle 
which is defined by $\psi=\mathrm{a} \tan (\tan \phi / \sin \alpha)$. The illustration of the hull angles in Lindqvist formulas is shown by Fig. 17.
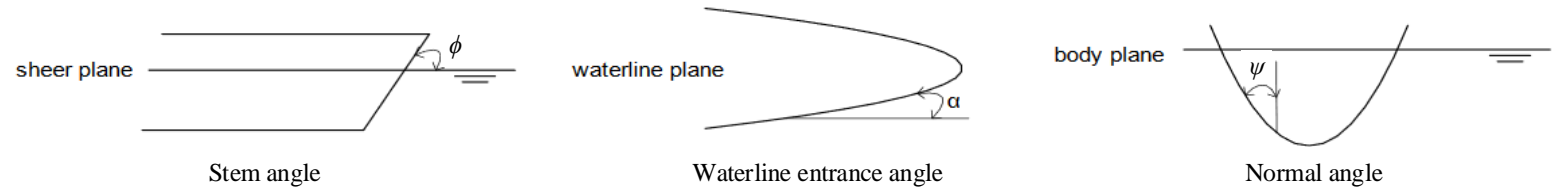

Fig. 17 Illustration of the hull angles in Lindqvist formula

The Riska semi-empirical formulas are given as:

$$
\begin{aligned}
& R_{i}=C_{1}+C_{2} V \\
& C_{1}=\frac{f_{1}}{\left(\frac{2 T}{B}+1\right)} B L_{P} h+(1+0.021 \phi)\left(f_{2} B h^{2}+f_{3} L_{B} h^{2}+f_{4} L_{B} h\right) \\
& C_{2}=(1+0.063 \phi)\left(g_{1} h^{1.5}+g_{2} B h\right)+g_{3} h\left(1+\frac{1.2 T}{B}\right) \frac{B^{2}}{\sqrt{T}}
\end{aligned}
$$

where $L_{P}$ and $L_{B}$ are length of parallel middle body and length of bow, which are $65 \mathrm{~m}$ and $39 \mathrm{~m}$ respectively. The other parameters are empirical constants, as given in Table 7.

Table 7 The constants used in Riska formula

\begin{tabular}{|c|c|c|c|c|c|c|c|}
\hline Items & $\begin{array}{c}f_{1} \\
\left(\mathrm{kN} / \mathrm{m}^{3}\right)\end{array}$ & $\begin{array}{c}f_{2} \\
\left(\mathrm{kN} / \mathrm{m}^{3}\right)\end{array}$ & $\begin{array}{c}f_{3} \\
\left(\mathrm{kN} / \mathrm{m}^{3}\right)\end{array}$ & $\begin{array}{c}f_{4} \\
\left(\mathrm{kN} / \mathrm{m}^{3}\right)\end{array}$ & $\begin{array}{c}g_{1} \\
\left(\mathrm{~m} / \mathrm{s}^{1.5} \mathrm{~m}^{1.5}\right)\end{array}$ & $\begin{array}{c}g_{2} \\
(\mathrm{~m} / \mathrm{s} \times \mathrm{m})\end{array}$ & $\begin{array}{c}g_{3} \\
\left(\mathrm{~m} / \mathrm{s}^{\times} \mathrm{m}^{2.5}\right)\end{array}$ \\
\hline Value & 0.23 & 4.58 & 1.47 & 0.29 & 18.9 & 0.67 & 1.55 \\
\hline
\end{tabular}

The ice resistances versus ship velocity obtained by numerical simulation and the semiempirical formulas are shown in Fig. 18, and the experimental results are also presented for the comparison. The ice resistance calculated by the two semi-empirical formulas increases linearly with the ship velocity, and the ice resistance calculated numerically also increases approximately linearly with the ship velocity. The ice resistance calculated by Riska formulas are significantly higher than the experimental results and other calculation results. This is because the influence of various ice properties on ice resistance is not considered in Riska formulas, which may influence the calculation accuracy of Riska formulas. The ice resistance calculated by Lindqvist formulas is smaller than the experimental results, especially for the scenarios with $950 \mathrm{~mm}$ thick ice. The similar result is also obtained by the experimental research by Myland and Ehlers [31]. The reason may be that the components of ice resistance due to the rotation of ice cusps is not taken into account in Lindqvist formulas. The ice resistance calculated by numerical simulation is closest to the experimental results. 


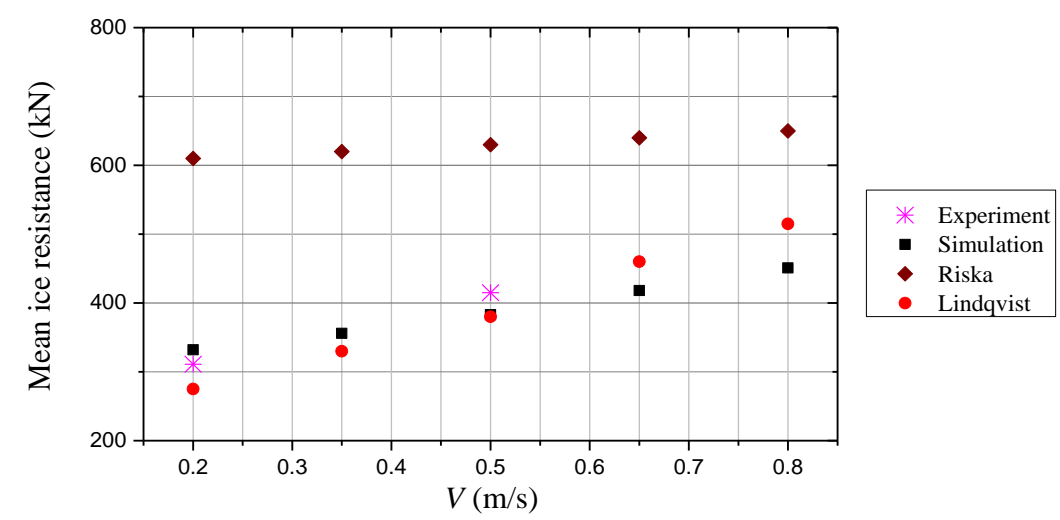

(a) $h=760 \mathrm{~mm}$

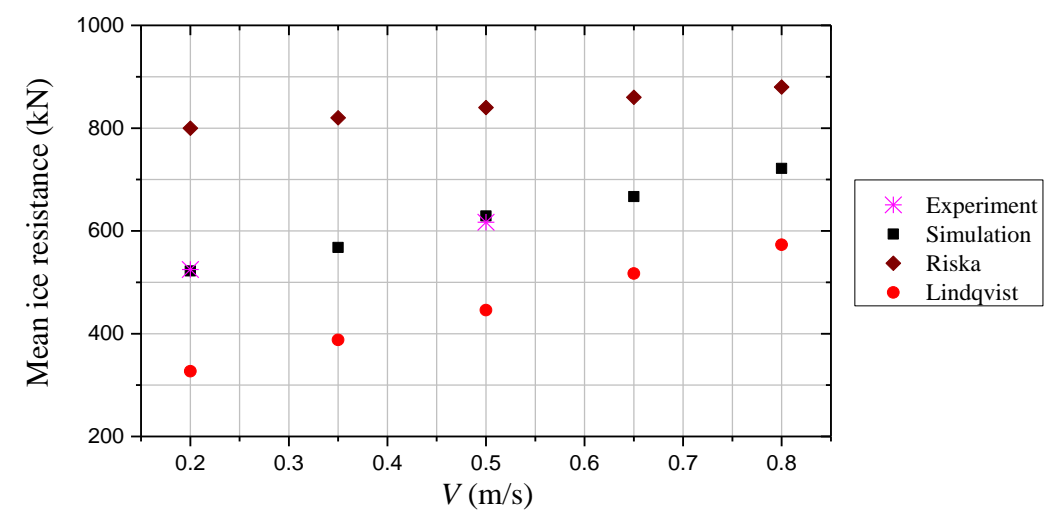

(b) $h=950 \mathrm{~mm}$

Fig. 18 The influence of ship velocity on mean ice resistance

Fig. 19 shows the relationship between the standard deviation of ice resistance and ship velocity calculated by the numerical simulations. It can be seen that the standard deviations of ice resistance present obvious decreasing trend with the increase of ship velocity. Especially when the ship velocity is greater than $0.5 \mathrm{~m} / \mathrm{s}$, the standard deviations of ice resistance decrease rapidly. Fig. 20 shows the time histories of longitudinal forces on ship with different velocities under two thick ice sheet. The force levels of ice resistance are higher at high velocity, which is because the impact between the ship and ice sheet at higher velocity will generate greater crushing contact force. However, the fluctuation amplitudes of ice resistance at high velocity are obviously smaller than those at low velocity. Fig. 21 shows the simulated results of continuous icebreaking process with different ship velocities. It can be observed that the width of broken ice channel at low velocity is obviously wider than that at high velocity. The width of broken ice channel at the velocity of $0.8 \mathrm{~m} / \mathrm{s}$ is only slightly wider than the ship breadth. This indicates that ice breaking length decreases rapidly at high velocity, which results in smaller fluctuation amplitude of ice resistance at high velocity.

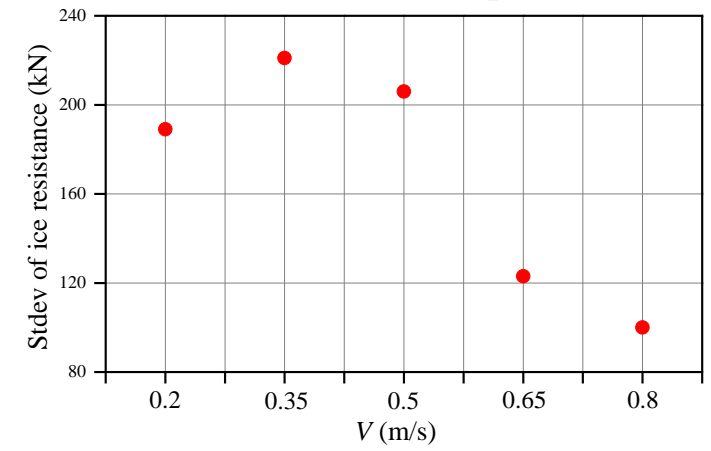

(a) $h=760 \mathrm{~mm}$

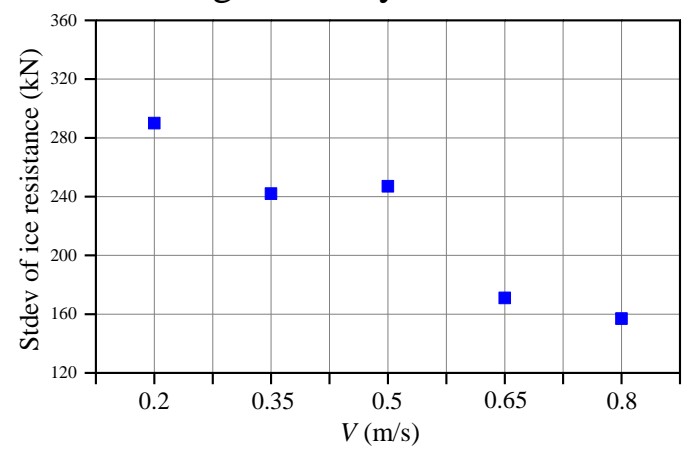

(b) $h=950 \mathrm{~mm}$

Fig. 19 The influence of ship velocity on standard deviations of ice resistance 
Study of continuous icebreaking process with cohesive element method
Feng Wang, Li Zhou, Zao-Jian Zou

Ming Song, Yang Wang, Yi Liu

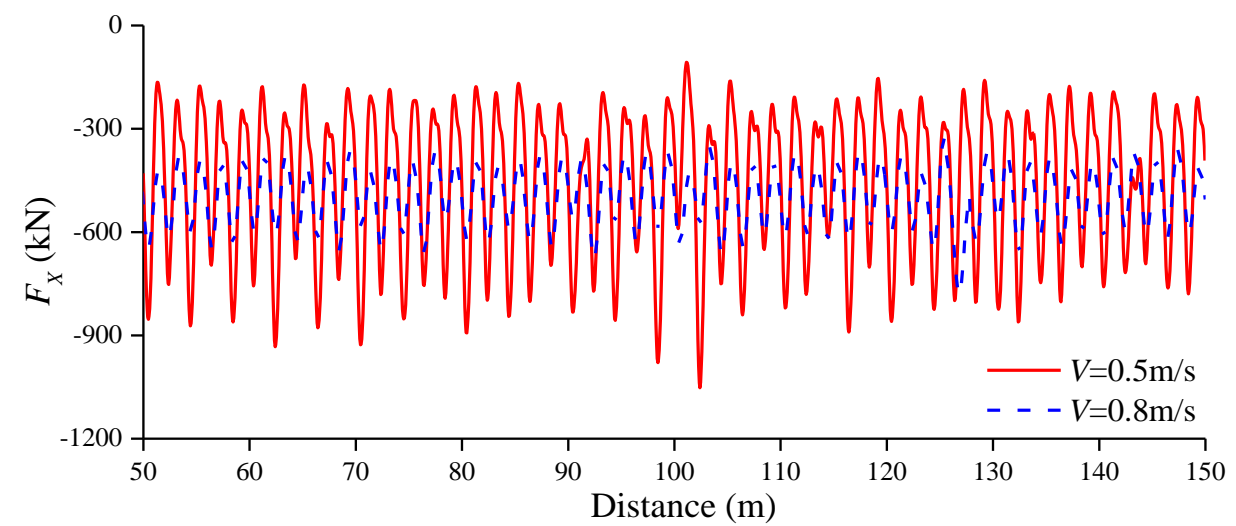

(a) $h=760 \mathrm{~mm}$

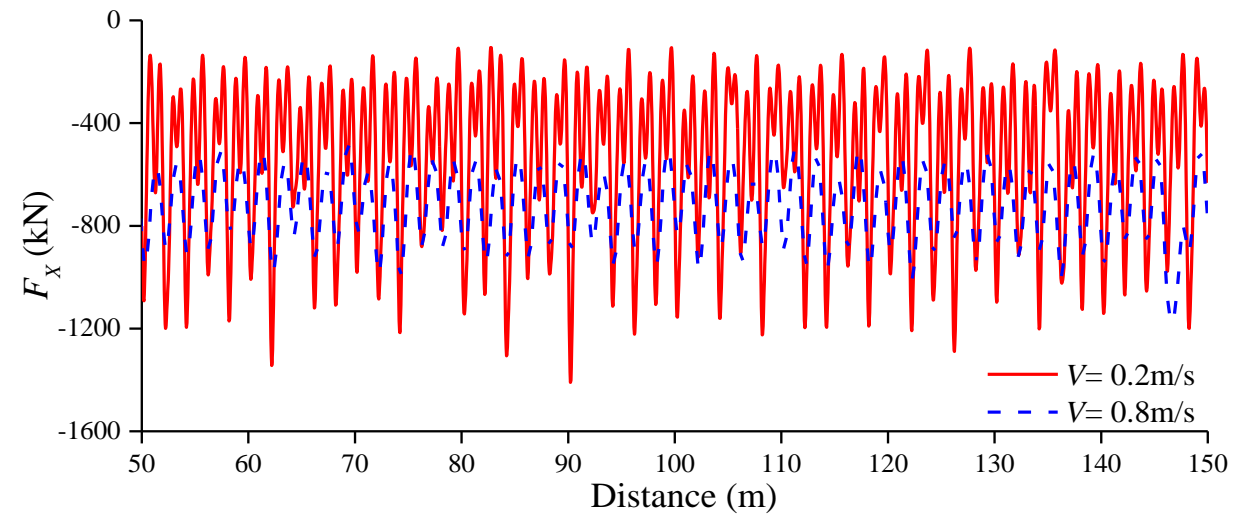

(b) $h=950 \mathrm{~mm}$

Fig. 20 The influence of ship velocity on longitudinal force
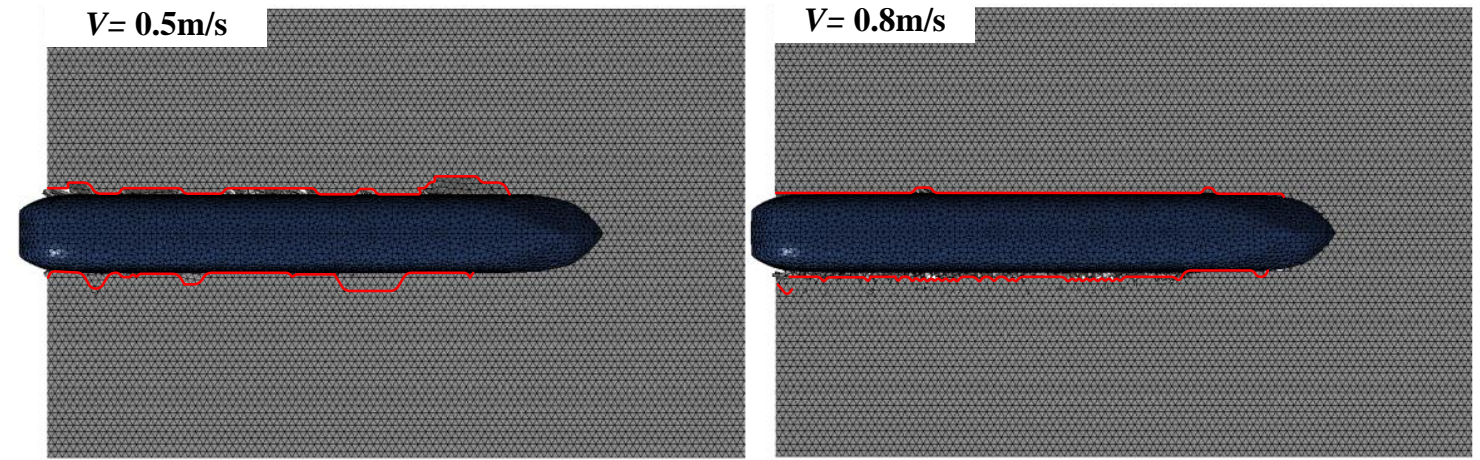

(a) $h=0.76 \mathrm{~m}$
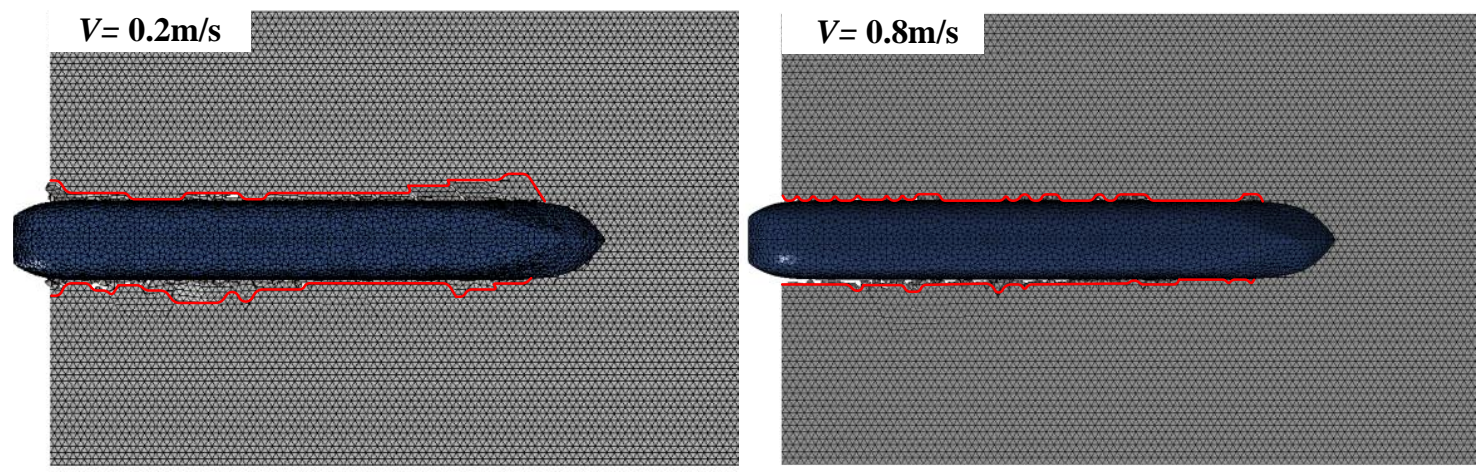

(b) $h=0.95 \mathrm{~m}$

Fig. 21 The simulated results of continuous icebreaking process with different ship velocities 
Feng Wang, Li Zhou, Zao-Jian Zou

Ming Song, Yang Wang, Yi Liu
Study of continuous icebreaking process

with cohesive element method

\section{Conclusion}

In this paper, the continuous icebreaking process in level ice for the icebreaking tanker MT Uikku is simulated by combining an elastoplastic softening constitutive model with cohesive element method (CEM). The numerical models are established in commercial finite element software LS-DYNA. Firstly, the elastoplastic softening constitutive model is calibrated by simulating ice cone crushing tests, and the effects of different softening laws on simulation results are evaluated. Then, the continuous icebreaking process is simulated by numerical methods. Mesh dependency study is performed and the simulated results are compared with the experimental results in terms of ice resistance and ice failure patterns. Finally, the effect of ship velocity on ice resistance and ice failure patterns are also analyzed and discussed. The main conclusions can be drawn as follows:

(1) Among three softening laws for the ice elastoplastic softening constitutive model, the numerical results calculated by linear softening law fits best with the experimental results in terms of force-displacement curves and pressure-area curves for the ice cone crushing tests.

(2) The correct selection of mesh size is crucial for the computational accuracy of the cohesive element method. With a mesh size similar to ice breaking length in the radial direction, the CEM simulation can provide a good estimation of ice resistance and modelling of ice failure patterns. The calculated longitudinal forces on the ship agree well with the measured ones in both of the time domain and frequency domain. The power spectral peaks of ice resistance obtained by numerical simulations and model tests are below $2 \mathrm{~Hz}$, which indicates that the ice bending-induced force with low frequency is dominant in the total ice resistance.

(3) The numerical method achieves good agreement with the experimental observations in terms of ice failure patterns. The simulations well reproduce the ice local crushing and bending failure, as well as the rotation, accumulation and sliding process of ice cusps during the continuous icebreaking process. The asymmetric and non-simultaneous failure patterns happened at the both sides of the hull cause an irregular icebreaking channel, which reflects the random and uncertain nature inherent in the continuous icebreaking process.

(4) The mean ice resistance calculated by simulations presents approximately linear increasing tendency with the increase of ship velocity. The numerical simulation results agree well with the experimental results. Riska formulas give the highest ice resistance prediction, whereas Lindqvist formulas underestimate ice resistance. The ice breaking length decreases rapidly with the increase of ship velocity, which results in the fluctuation amplitude of ice resistance to be obviously smaller at higher velocity.

It is shown that the presented numerical methods capture well the main features of the continuous icebreaking process and have a good prospect in modeling ship-ice interactions. In order to facilitate the comparison between numerical results and available experimental results, two kinds of ice with different properties are established in the calibration of ice constitutive model and validation of numerical methods to simulate ship icebreaking process. Different ice properties may generate deviations on the numerical results. However, limited by the available experimental data, the possible deviation arisen from this could not be taken into consideration. In addition, the above conclusions are drawn based on the present simulations on the continuous icebreaking process of the icebreaking tanker - MT Uikku. The reliability and generality of the numerical results still need to be proved by more investigations on other ships in the future. 


\section{ACKNOWLEDGEMENTS}

The corresponding author (second author) greatly acknowledges the supports of the National Natural Science Foundation of China (Grant No. 51809124, 5181102016, 51709136), Natural Science Foundation of Jiangsu Province of China (Grant No. BK20170576), the Natural Science Foundation of the Higher Education Institutions of Jiangsu Province of China (Grant No. 17KJB580006) and State Key Laboratory of Ocean Engineering (Shanghai Jiao Tong University) (Grant No. 1704).

\section{REFERENCES}

[1] Croasdale, K.R. and Cammaert, A.B., 1994. An improved method for the calculation of ice loads on sloping structures in first-year ice. Hydrotechnical Construction, 28(3), 174-179. https://doi.org/10.1007/BF01545935

[2] Enkvist, E., 1972. On the ice resistance encountered by ships operating in the continuous mode of icebreaking. Report No. 24, Swedish Academy of Engineering Sciences in Finland, Helsinki, Finland.

[3] Lewis, J. W., DeBord, F. W. and Bulat, V. A., 1982. Resistance and propulsion of ice-worthy ships. Transactions-Society of Naval Architects and Marine Engineers, 90, 249-276.

[4] Keinonen, A. J., Browne, R., Revill, C. and Reynolds, A., 1996. Icebreaker characteristic synthesis, Report TP12812E, Transportation Development Centre, Calgary, Canada.

[5] Lindqvist, G., 1989. A straightforward method for calculation of ice resistance of ships. Proceeding of 10th International Conference on Port and Ocean Engineering under Arctic Conditions, Lulea, Sweden, 722-735.

[6] Lindstrom, C. A., 1990. Numerical estimation of ice forces acting on inclined structures and ships in level ice. Proceeding of 22nd International Offshore Technology Conference, Houston, USA. https://doi.org/10.4043/6445-MS

[7] Riska, K., Wilhelmson, M., Englund, K. and Leiviskä, T., 1997. Performance of merchant vessels in ice in the Baltic. Report 52, Winter Navigation Research Board, Helsinki, Finland.

[8] Paavilainen, J., Tuhkuri, J. and Polojärvi, A., 2006. Discrete element simulation of ice pile-up against an inclined structure. Proceedings of 18th International Symposium on Ice, Sapporo, Japan, 177-184.

[9] Zhan, D., Agar, D., He, M., Spencer, D. and Molyneux, D., 2010. Numerical simulation of ship maneuvering in pack ice. Proceeding of 29th International Conference on Offshore Mechanics and Arctic Engineering, Shanghai, China, 855-862. https://doi.org/10.1115/OMAE2010-21109

[10] Wang, B., Yu, H. C. and Basu, R., 2008. Ship and ice collision modeling and strength evaluation of LNG ship structure. Proceeding of 27th International Conference on Offshore Mechanics and Arctic Engineering, Estoril, Portugal, 911-918. https://doi.org/10.1115/OMAE2008-57134

[11] Liu, Z., Amdahl, J. and Løset, S., 2011. Integrated numerical analysis of an iceberg collision with a foreship structure. Marine Structures, 24(4), 377-395. https://doi.org/10.1016/j.marstruc.2011.05.004

[12] Shi, C., Hu, Z. and Luo, Y., 2016. An elastic-plastic iceberg material model considering temperature gradient effects and its application to numerical study. Journal of Marine Science and Application, 15(4), 370-375. https://doi.org/10.1007/s11804-016-1384-4

[13] Paavilainen, J., Tuhkuri, J. and Polojärvi, A., 2009. 2D combined finite-discrete element method to model multi-fracture of beam structures. Engineering Computations, 26(6), 578-598. https://doi.org/10.1108/02644400910975397

[14] Paavilainen, J., Tuhkuri, J. and Polojärvi, A., 2011. 2D numerical simulations of ice rubble formation process against an inclined structure. Cold Regions Science and Technology, 68(1), 20-34. https://doi.org/10.1016/j.coldregions.2011.05.003

[15] Hilding, D., Forsberg, J. and Gürtner, A., 2011. Simulation of ice action loads on offshore structures. Proceeding of 8th European LS-DYNA Users Conference, Strasbourg, France, 1-12.

[16] Hillerborg, A., Modéer, M. and Petersson, P.E., 1976. Analysis of crack formation and crack growth in concrete by means of fracture mechanics and finite elements. Cement and Concrete Research, 6(6), 773781. https://doi.org/10.1016/0008-8846(76)90007-7

[17] Mulmule, S.V. and Dempsey, J.P., 1997. Stress-separation curves for saline ice using fictitious crack model. Journal of Engineering Mechanics, 123(8), 870-877. https://doi.org/10.1061/(ASCE)07339399(1997)123:8(870) 
[18] Mulmule, S.V. and Dempsey, J.P., 2000. LEFM size requirements for the fracture testing of sea ice. International Journal of Fracture, 102(1), 85-98. https://doi.org/10.1023/A:1007603428907

[19] Gürtner, A., 2009. Experimental and numerical investigations of ice-structure interaction. Ph.D thesis, Department of Civil and Transport Engineering, NTNU, Trondheim, Norway.

[20] Konuk, I. and Yu, S., 2010. A cohesive element framework for dynamic ice-structure interaction problems: Part III-Case studies. Proceeding of 29th International Conference on Offshore Mechanics and Arctic Engineering, Shanghai, China, 801-809. https://doi.org/10.1115/OMAE2010-20577

[21] Lu, W., Lubbad, R. and Løset, S., 2014. Simulating ice-sloping structure interactions with the cohesive element method. Journal of Offshore Mechanics and Arctic Engineering, 136(3), 031501. https://doi.org/10.1115/1.4026959

[22] Wang, F., Zou, Z. J., Zhou, L., Ren, Y. Z. and Wang, S. Q., 2018. A simulation study on the interaction between sloping marine structure and level ice based on cohesive element model. Cold Regions Science and Technology, 149, 1-15. https://doi.org/10.1016/j.coldregions.2018.01.022

[23] Wang, F., Zou, Z. J., Li, Z., Wang, Y., Yu, H., \& Zhang, H., 2019. Numerical simulation of ice milling loads on propeller blade with cohesive element method. Brodogradnja, 70(1), 109-128. https://doi.org/10.21278/brod70108

[24] Dempsey, J.P., Xie, Y., Adamson, R.M. and Farmer, D.M., 2012. Fracture of a ridged multi-year Arctic sea ice floe. Cold Regions Science and Technology, 76, 63-68. https://doi.org/10.1016/j.coldregions.2011.09.012

[25] Timco, G. W. and Weeks, W. F., 2010. A review of the engineering properties of sea ice. Cold Regions Science and Technology, 60(2), 107-129. https://doi.org/10.1016/j.coldregions.2009.10.003

[26] Cornec, A., Scheider, I. and Schwalbe, K.H., 2003. On the practical application of the cohesive model. Engineering Fracture Mechanics, 70 (14), 1963-1987. https://doi.org/10.1016/S0013-7944(03)00134-6

[27] Kim, H., Daley, C. and Colbourne, B., 2015. A numerical model for ice crushing on concave surfaces. Ocean Engineering, 106, 289-297. https://doi.org/10.1016/j.oceaneng.2015.07.020

[28] Masterson, D. M., Frederking, R. M. W., Wright, B., Kärnä, T. and Maddock, W. P., 2007. A revised ice pressure-area curve. Proceeding of 19th International Conference on Port and Ocean Engineering under Arctic Conditions, Dalian, China, 305-314.

[29] Zhou, L., Riska, K., und Polach, R. V. B., Moan, T. and Su, B., 2013. Experiments on level ice loading on an icebreaking tanker with different ice drift angles. Cold Regions Science and Technology, 85, 79-93. https://doi.org/10.1016/j.coldregions.2012.08.006

[30] Zhou, L., Riska, K., Moan, T. and Su, B., 2013. Numerical modeling of ice loads on an icebreaking tanker: Comparing simulations with model tests. Cold Regions Science and Technology, 87, 33-46. https://doi.org/10.1016/j.coldregions.2012.11.006

[31] Myland, D. and Ehlers, S., 2016. Influence of bow design on ice breaking resistance. Ocean Engineering, 119: 217-232. https://doi.org/10.1016/j.oceaneng.2016.02.021

Submitted: 18.06.2019. Feng Wang, wangfeng0630@hotmail.com

Accepted: $\quad 17.072019 \quad$ School of Naval Architecture, Ocean and

Li Zhou, zhouli209@ hotmail.com (Corresponding Author)

Jiangsu University of Science and Technology, Zhenjiang 212003, China

Zao-Jian Zou, zjzou@sjtu.edu.cn

School of Naval Architecture, Ocean and Civil Engineering, Shanghai Jiao

Tong University, Shanghai 200240, China

Collaborative Innovation Center for Advanced Ship and Deep-Sea

Exploration, Shanghai Jiao Tong University, Shanghai 200240, China

Ming Song, songmingcssc@163.com

Jiangsu University of Science and Technology, Zhenjiang 212003, China

Yi Liu, lyly0309@126.com

School of Naval Architecture, Ocean and Civil Engineering, Shanghai Jiao

Tong University, Shanghai 200240, China

Yang Wang, maible@sjtu.edu.cn

School of Naval Architecture, Ocean and Civil Engineering, Shanghai Jiao

Tong University, Shanghai 200240, China 